\title{
A qualitative study to explore the help seeking views relating to depression among older Black Caribbean adults living in the UK
}

Natalie Victoria Bailey

A thesis submitted in partial fulfilment of the requirements of the University of East London for the degree of Professional Doctorate in Counselling Psychology.

Student No: 1137793

Resubmission: July 2020 


\begin{abstract}
Rationale: Worldwide, literature has documented a rise in number among the older adult population, and a high prevalence of depression within this group. In the UK, rates of mental health problems within Black and minority groups are thought to be higher than in the white population. Mental health service providers need to understand the experiences and beliefs that underlie help seeking behaviour in order to deliver effective, culturally appropriate and accessible services; however, literature suggests these beliefs have rarely been explored within older adults from ethnic minority groups. Aims: Research specifically on help seeking among Black Caribbean people is limited. Therefore, this study will explore the personal experiences, meanings, and contexts that underlie help seeking in relation to self-defined depression among the UK's older Black Caribbean adults, both now and when younger. In addition, the study aims to explore some of the barriers experienced by participants, if any when considering seeking help from mental health services for depression.

Method: Semi-structured interviews were conducted with 8 UK Black Caribbean participants, aged between 65-79 years. Transcripts were analysed us ing Interpretative Phenomenological Analysis principles.

Findings: Three master themes emerged from analys is: 1) "If you don't know, you don't seek help" 2) “....I was depressed...I knew I was depressed" and 3) "You have to decide": Attitudes to help seeking and mental health service use. A description of these Master themes and ten related subordinate themes are discussed.

Conclusion: The findings revealed how participants past personal experiences of coping with depression including migratory histories, cultural and religious views and personal relationships influenced help seeking views and preferred coping methods for depression. In addition, several issues were discovered to influence decision-making on whether to seek help or not from mental health services for depression. Findings from this study could be considered to inform the delivery of mental health services and be transferred into policy.
\end{abstract}

Key words: Older Adults, Black Caribbean, Help Se eking, Depression 


\section{Table of Contents}

Abstract

Table of Contents

Acknowledgements vi

Author's Personal Reflections vii

CHAPTER ONE

1 Introduction 1

$\begin{array}{lll}1.1 & \text { Statistics on the Older Adults' Population } & 1\end{array}$

1.2 Statistics on Depression Later on in Life 1

1.3 Ethnic Minority Groups and Depression in the UK 2

1.4 The Need to Study Help Seeking Views among the UK's Black Caribbean

Older Adults 3

1.5 Contribution to Counselling Psychology 3

1.6 Scope and Aims of Study 4

1.7 Researcher's Reflexivity 5

\section{CHAPTER TWO}

2 Literature Review

2.1 Ageing

2.2 Depression and Older People $\quad 7$

2.3 Older Adults Mental Health Service Use 9

2.4 Ethnic Minority Older Adults' Accessing Mental Health Services 11

2.5 Help Seeking Theories 13

2.6 Older Adults, Coping and Help Seeking 13

2.7 Black Caribbean Older Adults, Coping and Help Seeking 14

2.8 Culture and Help Seeking 16

2.9 Older Adults and Health professionals 17

$\begin{array}{ll}2.10 \text { The Relational Preferences of Service Users } & 18\end{array}$

\section{CHAPTER THREE}

3 Methodology 20

3.1 Chapter Three Overview 20

3.2 Rationale for Qualitative Research 20

3.3 Rationale for Research Paradigm 21

3.3.a Positivism Paradigms $\quad 22$

3.3.b Post-Positivism Paradigms 22

3.3.c Interpretivist-Constructivist Paradigm 22

$\begin{array}{ll}3.4 \text { Rationale for a Critical Realist Position } & 23\end{array}$

3.5 Rationale for Interpretative Phenomenological Analysis (IPA) 23

3.6 Explanation of the Study's Design 25

3.6.1 Data Collection 25

3.6.2 Construction of Interview Schedule 25

3.7 Criteria for the Inclusion and Exclusion of Participants 26

$\begin{array}{ll}\text { 3.7.1 Recruitment Procedure } & 26\end{array}$

$\begin{array}{ll}\text { 3.7.2 Sampling Criteria } & 27\end{array}$

3.7.3 The Sample 28 
Table One (Depiction of Sample) 28

3.8 Interview Procedure 28

3.9 Data Examination 29

3.9.1 Steps of Examination $\quad 29$

3.10 IPA's Quality and Accuracy Process 30

3.10a Sensitivity to Context 31

3.10b Commitment and Rigour 31

3.10c Coherence and Transparency $\quad 32$

3.10d Impact and Importance $\quad 32$

3.11 Ethical Considerations 32

\section{CHAPTER FOUR}

4. Results

4.1Chapter Four Overview 34

Table 2 Master Themes and Subordinate Themes 35

4.2 Overview of Master Theme One 36

4.2.1 Participants' Experiences of Migration in relation to Depression 36

$\begin{array}{ll}\text { 4.2.2 "Just get on with it" } & 38\end{array}$

4.2.3 Participants' Significant Relationships Experiences in relation to
Depression

4.2.4 Participants' Experiences of Health Professionals \& Listening Skills 43

4.3 Overview of Master Theme Two 45

4.3.1 Grief in Later Life and Depression 45

4.3.2 A matter of "Inconvenience" 47

4.4 Overview of Master Theme Three 48

4.4.1 "..Recognise and Start ..." 49

4.4.2 "Why should I go tell somebody my business?" 50

4.4.4 "No guarantee": Professional services 53

4.4.5 "Just get on with it" part 2

4.5 Summary of Results Chapter 56

\section{CHAPTER FIVE}

5 Discussion $\quad 58$

5.1 Chapter Five Overview $\quad 58$

5.2 Reflection of the Study's Aims

5.2.1 Overview of Key Findings 58

5.3 Research Question One $\quad 59$

5.3.1 "If you don't know, you don't seek help"

5.3.2 Coping with Depression $\quad 59$

5.3.3 Reactive Depression vs Endogenous Depression 60

5.3.4 Participants' Experiences of Migration in relation to Depression 60

5.3.5 The Influence of Culture on Coping in relation to Depression 61

5.3.6 The Influence of a Self-Sufficient Outlook on Coping in relation

to Depression $\quad 62$

5.3.7 Participants' Significant Relationships Experiences in relation
to Depression

5.3.6 Stigma and Beliefs in relation to Depression 63

5.3.7 Participants' Experiences of Health Professionals and Listening Skills 63

5.4 “...I was depressed...I knew I was depressed" 64 
5.4.1 Coping with Depression Aged 65 Years and Over 64

5.4.2 Grief in Later Life and Coping in relation to Depression 64

5.4.3 Religion, Spirituality, Faith and Coping in relation to Depression 64

5.4.4 Shared Experiences and Coping in relation to Depression 65

5.4.5 A Matter of "Inconvenience" 65

5.5 Research Question Two 65

5.5.1 "You have to decide": Attitudes to Help Seeking and Mental Health

$\begin{array}{ll}\text { Service Use. } & 66\end{array}$

5.5.2 A "First Step" to Help Seeking in relation to Mental Health Services

for Depression 66

5.5.3 Influence of Significant Relationships on Help Seeking in relation to

Mental Health Services for Depression $\quad 66$

5.5.4 Stigma, Beliefs and Culture on Help Seeking in relation to Mental Health

$\begin{array}{ll}\text { Services for Depression } & 67\end{array}$

5.5.5 A Matter of 'Choice'

5.5.6 The influence of Language on Help Seeking in relation to Mental Health $\begin{array}{ll}\text { Services for Depression } & 68\end{array}$

5.5.7 Previous Experience with Health Professionals in relation

to Depression

68

5.5.8 The Influence of Childhood on Help Seeking in relation to Mental Health

Services for Depression

5.6 Implication for Counselling Psychology and Clinical Practice 69

$\begin{array}{ll}\text { 5.6a Service level } & 69\end{array}$

$\begin{array}{ll}\text { 5.6b Clinical level } & 72\end{array}$

$\begin{array}{ll}\text { 5.7 Strengths and Limitations } & 73\end{array}$

5.8 Conclusions and Recommendations for Future Research 76

$\begin{array}{ll}\text { REFERENCES } & 78\end{array}$

$\begin{array}{ll}\text { Appendix A Interview Schedule } & 90\end{array}$

Appendix B Information Sheet/Letter 92

Appendix Ba Email Recruitment Example 1 94

Appendix Ba Email Recruitment Example 2 95

Appendix Ba Email Recruitment Example 3 96

Appendix Ba Email Recruitment Example 4 97

Appendix C Consent Form $\quad 98$

Appendix D Debrief form $\quad 99$

$\begin{array}{ll}\text { Appendix E Ethical Approval } & 100\end{array}$

$\begin{array}{ll}\text { Appendix F Change of Title 1 } & 104\end{array}$

$\begin{array}{ll}\text { Appendix G Example of Emerging Themes } & 105\end{array}$

$\begin{array}{ll}\text { Appendix H Examination of Data Process Example 1 } & 106\end{array}$

$\begin{array}{ll}\text { Appendix I Examination of Data Process Example } 2 & 107\end{array}$

Appendix J Examination of Data Process Example $3 \quad 108$

Appendix K Examination of Data Process Example 4 109 


\section{Acknowledgements}

My heavenly Father, my creator, my God, I could not have completed this journey without your grace and love. You provided for all my needs. I honour you and publicly give you thanks and praise.

\section{Dedication}

This thesis is dedicated to my beloved parents Vilna Louise Cruickshank and Uriah Bailey, who both sadly passed away during my training on the professional doctorate programme in Counselling Psychology. I honour their memory by successfully completing this thes is and in turn this doctoral programme. I will forever remain grateful for their love, encouragement and belief in me. Thank you mummy and daddy. We did it!

I also dedicate this thesis to my daughter Gabrielle, who now wants to pursue a career in psychology after observing me on this journey. Gabrielle, whatever you decide to do, just know that I love you, support you and that I am extremely proud of you. Thank you for your patience, support and encouragement along this journey. We did it!

\section{Special Thanks}

Thank you to my twin sister Natasha and my niece Amarachi. Our household has worked as a team. I am proud of us! Together, we have all made sacrifices so that I am where I am today. Thank you for your support, encouragement and love. We did it!

Thank you to my supervisor Professor Rachel Tribe, your guidance, support and understanding will never be forgotten. Also, a special thank you to all the participants in this study. It is an honour to share your voice with the world.

There are so many people I have to thank for being there for me along this journey to the finishing line; however, limited space permits me from naming you all. Therefore, a collective thank you is offered to all for your encouraging words, prayers, support, guidance, love, understanding and patience. You all played a role in me getting here.

\section{\#Neverforgotten\#Alwaysgrateful}




\section{The Author's Personal Reflections}

The experience of conducting research on help seeking views relating to depression among Black Caribbean adults living in the UK was thought provoking and fascinating for me. What particularly felt quite touching and remained strongly with me was learning about participant's experiences arriving to UK for the first time as migrants, and how this influenced the ir thoughts and feelings of depression. With further reflection, I realised just how much of my personal history I was not aware of (being of Black Caribbean decent) and felt a profound appreciation for the evident effort my parents made to protect me from experiencing what they had experienced when first arriving to the UK.

As I analysed participants' accounts, I recognised similarities in attitudes and values held within my own family and upbringing. For example, my parents (now both sadly passed away in recent years) had experiences similar to those of the participants in my research, they were given the "opportunity" to come to the UK to "better themselves", and in turn improve the lives of their children. I recognised that they achieved this and subsequently I remain forever grateful.

Importantly,Willig and Stainton-Rogers, (2008) notes how the researcher's own identity, values and interests unavoidably impacts on the research process. For this reason, the interpretation of the analysis in this study, to some extent will almost certainly be coloured by my own experiences. Nevertheless, a voice was given to a population, who is underexplored, and in turn contributes further to know ledge on older Black Caribbean adults' experiences and beliefs that underlie help seeking behaviour, in order to deliver effective, culturally appropriate and accessible services. With that said, overall it felt important to me to honour participants by ensuring their experiences were heard and understood. I am grateful to be part of the journey in achieving this. 


\section{CHAPTER ONE: INTRODUCTION}

\subsection{Statistics on the Older Adults' Population}

The world's population of older adults has never been as high as it is today (The World Population Prospects, 2019). It has been predicted that by 2050, one in six people in the world will be aged 65 and over; and in Europe and Northern America, one in four persons could be aged 65 or over. Specific to the UK, the Office of National Statistics (ONS), 2019 documented an increase by 47 per cent in the number of older adults aged 65 and over, since 1974. Furthermore, people in the UK aged 65 years and older are more in number than those aged less than 16 years (ONS, 2019).

In addition, worldwide people aged 80 years or over, is projected to triple, from 143 million in 2019 to 426 million in 2050 (The World Population Prospects, 2019). Therefore, as the older adult population is evidently rising in number, there is a wealth of lived experiences waiting to be further explored and understood - specifically, gaining a greater understanding of concerns surrounding mental health and the older adult population. This understanding could then be used to improve service provision in the future.

\subsection{Statistics on Depression Later on in Life}

Among older people depression has been identified as a mental health condition that is commonly experienced, affecting 10-15 per cent of the population (Baldwin, 2014; Huang and Carpenter, 2011; Conner et al., 2010). Similarly, the World Health Organisation (WHO), 2016 reported depression as a common condition among those aged 65 and above. With these findings, and with an evident ageing population, prospectively, there is concern about the rise of depression among older adults.

NHS England (2014) identified a notable relationship between older adults, mental health issues, depression and treatment outcomes. When examining 1000 individuals aged over 65 years, they found 250 will experience a mental difficulty, of which 135 will experience depressive symptoms, and 115 will not obta in treatment. The report also identified 85 per cent of older adults with depressive symptoms did not obtain any help specifically from the NHS. 
Interestingly, WHO, (2016) reported that only one third of older adults with depressive symptoms requiring intervention, will have a conversation with their GP about their symptoms. What is more, for those who decide to have this conversation, only half will receive treatment. WHO, (2016) further reported the treatment received as primarily medication based; nevertheless, although medication base treatments are helpful, the National Institute for Health and Clinical Excellence (NICE) guiding principles suggest a wide variety of treatments, including Psychological therapy, to be obtainable for older people with depression (Tribe, 2017).

Mortimer and Green (2016) also identified significant concerns with older adults' mental health. Depression as well as other mental health conditions was reported to often not be acknow ledged or diagnosed among older adults. Moreover, 50 per cent of younger individuals (with depressive symptoms) compared to 6 per cent of older adults (with depressive symptoms) are being referred to wellbeing and mental health services. Recognising and identifying these gaps in service provision among older adults with depression, not only allows for a wealth of lived experience to be further discovered and understood; it can also facilitate further improvement of service provision.

\subsection{Ethnic Minority Groups and Depression in the UK}

When considering the data collated on mental health in ethnic minority groups, it is important to note that sample sizes are often small which can limit our capacity to look overall at mental health among this population group (Mental Health Foundation (MHF), 2019). Often it is difficult to get accurate prevalence rates of mental health problems because of sampling tools, measurements used, the hidden nature of mental health issues and underfunding; for these reasons, making direct comparisons can be problematic at times (Mental Health Foundation , 2016). Given these limitations, there is still much to learn and understand about ethnic minority communities and mental health in the UK (MHF, 2019). What is more, as people from these ethnic minority communities often face individual and societal challenges that can affect access to all healthcare services, the importance of gaining a deeper understanding of mental health concerns among ethnic communities in the UK becomes even more apparent (MHF, 2019).

Previous research in the UK has identified some ethnic minority groups as having a higher percentage of depression compared to the white population (Mental Health Foundation, (MHF) 2007; National Service Frameworks, 1999). Similarly, some rates 
of mental health problems within ethnic minority groups in the UK are also thought to be higher than in the white population (MHF, 2019; Stevenson and Rao, 2014; MHF, 2007; National Institute for Mental Health in England, 2003). However, given the challenges with methodological approaches used for research in this area, these findings cannot be seen as representative of all ethnic minority communities, and suggests overall the importance for further research in relation to depression and other mental health difficulties among this population. In doing so, mental health services can work towards providing effective culturally located and appropriate access to service users from all communities (Williams et al., 2015).

\subsection{The Need to Study Help Seeking Views among the UK's Black Caribbean Older Adults}

People of Black Caribbean origin in the UK, form one of the longest and largest established ethic minority populations (Williams et al., 2015). Importantly, as most migration occurred in the 1950's and 60's, the first-generation of migrants in the UK are now reaching retirement age. As a result, the need for focused investigation of this population's particular mental health concerns arises (McIntosh and Huq, 2017; Williams et al., 2015).

In addition, research on help seeking among people of Black Caribbean origin is limited (Woodward et al., 2013). Whilst numerous studies have examined barriers to treatment amongst older people, there is a scarcity of qualitative studies that examine the experiences of Black Caribbean older adults and their views of barriers faced when looking for help from wellbeing and mental health services. The wealth of data accessible through qualitative analysis can enhance the critical depth to existing quantitative studies in the field. Furthermore, such qualitative research may highlight aspects that influence the process of help seeking among the older Black Caribbean population in the UK.

\subsection{Contribution to Counselling Psychology}

Counselling Psychologists are in a position to better understand the lived experience of older Black Caribbean adults - looking at each individual in a pluralistic way. Qualitative research is considered best suited to understand individual differences, meanings and contexts (Yardley, 2000; Rennie, 2007); however, as noted earlier there is 
a scarcity of qualitative studies that examine the experiences of Black Caribbean older adults and their views of barriers faced when looking for help from wellbeing and mental health services. The contribution of gaining further knowledge from older Black Caribbean adults in this study (from a qualitative approach, see chapter 3 for a detailed explanation), could potentially aid Counselling Psychologis ts and other mental health professionals to plan collaboratively with service users to develop more effective, culturally appropriate and accessible services, to ensure the needs of this increasing populace are met.

The values of Counselling Psychology are embedded in humanistic and existential phenomenological principles (BPS Division of Counselling Psychology, 2014); furthermore, Counselling Psychologists aim to work with the individual's subjective or inter-subjective experiences, in order to empower the individuals' steps in selfdevelopment. With that said, the prioritisation of Black Caribbean older adults' subjective or inter-subjective psychological experiences with depression could further help Counselling Psychologists and other mental health professionals learn how these experiences manifest or not in behaviour; as well as learn what this population do to cope in such instances.

On the whole, the values of Counselling Psychology are cons istent with the aims of this study, which is to explore and contextualise the UK's older Black Caribbean adults' experiences of coping with depression - specifically, exploring the ir help seeking views ,perceptions and beliefs with what will help someone with depression. Counselling Psychology is a fairly new discipline compared to other disciplines in psychology. Therefore, there is great opportunity to contribute knowledge to Counselling Psychology by conducting or iginal research grounded within the discipline's theoretical concerns.

\subsection{Scope and Aims of Study}

The importance of listening to communities and community organisations is being increasingly recognised as part of a process that can reduce health inequalities (WHO, 2020), enhance the quality of services (NICE, 2016) and contribute to enhancing accessibility and appropriateness (British Psychological Society, 2018). Mental health providers need to comprehend the decisions and experiences of service users or potential service users in order to understand and plan appropriate service provision, and to deliver effective, culturally appropriate and accessible services. What is more, 
this has particular resonance when groups which have been defined as hard to reach are considered (Tribe \& Bell, 2018).

Given these reasons above, this study will explore the personal experiences, meanings, and contexts that underlie help seeking in relation to self-defined depression among the UK's older Black Caribbean adults, both now and when younger. In addition, the study aims to explore some of the barriers experienced by participants, if any when considering seeking help from mental health services for depression. In line with these objectives, the research questions developed are:

1) How do older Black Caribbean adults make sense of coping with (self-defined) depression?

2) How can we better understand the decisions that older Black Caribbean adults make surrounding help seeking and accessing mental health services in relation to depression?

\subsection{Researcher's Reflexivity}

Reflexivity is a distinct feature of qualitative research (Banister et al., 1994); moreover, it entails a crucial reflection of self; meaning, the researcher acknowledges the impact of their own personal background, beliefs and behaviour on the research process (Finlay and Gough, 2003). Therefore, in reflexivity not only is the participant included in the construction of knowledge process, but so is the researcher (Fade, 2004). Furthermore, reflection of self or self-deliberation is crucial to comprehending how meanings are described and interpreted within Interpretative Phenomenological Analys is (IPA) (see chapter 3 for detailed explanation of IPA).

The basis of my reflexivity stems from the knowledge that Black and ethnic minority psychologists are underrepresented in the UK mental health services (BPS BAME representation and psychology, 2020; ONS, 2018; Maxie et al., (2006); Hays and Iwamasa, 2006; Hays, 2001). In addition, my reflexivity also holds in mind the knowledge that qualitative research within the Black Caribbean community is also limited (Woodward et al., 2013).

Being of Black Caribbean decent, I wanted to conduct a piece of research that related to the Black Caribbean community. I feel sharing this identity will potentially help to bridge the gap in research, and perhaps encourage a genuine openness with participants 
when sharing their experiences of coping with depression; as well as with expressing their views/attitudes towards helping seeking and mental health services in the UK. For these reasons, I feel I am in a position to make a positive contribution towards the field as a Black Caribbean trainee Counselling Psychologist - exploring a population that is not only underrepresented in the profession but also within qualitative research.

Growing as a reflective practitioner, involves a process whereby trainees and clinicians can explore the ir own beliefs, frames of reference, the language that is used that carries its own meaning, and how this informs their own research or clinical work (Tribe and Morrissey, 2015). Given this process, I understand that being a trainee Counselling Psychologist of Black Caribbean decent may sway what participants will share with me - meaning how their experiences are talked about, the knowledge formed and the explanations made. Furthermore, I am aware that these aspects may influence my interpretation of the narratives explored. As a result, I maintained regular supervision and kept a journal to remain mindful of these influences throughout the process. 


\section{CHAPTER TWO: LITERATURE REVIEW}

\subsection{Ageing}

The increasing longevity of our modern world's population, promotes further research (across disciplines), to better understand its impact and significance today. Recent demographic predictions estimate a continuous rise in the number of older people, and that people who are categorised as very old ( 80 years and older) will rise even faster (Vanleerberghe et al., 2017).

People are living longer due to advances in medicine and technology; longevity also occurs due to better living conditions (Vanleerberghe et al., 2017). As our world's population are living longer, it is critical for reflection on legislation relating to the rights and values of older adults requiring a variety of new services or adaptions to current services; this is to ensure the growing demand on health services (including mental health and social care) are met (McIntosh and Huq 2017). In addition, this growth requires health professionals to develop better strategies of engagement with this population and among and between services.

The ageing process may also bring about various psychological dilemmas and challenges. Thoughts surrounding end of life, achievements and decisions made (with opportunities taken or not) can influence significant psychological change during this stage (Tribe, 2017). For many older adults, these factors (including others) can affect their sense of self and well-being; as well as, amplify feelings of uncertainty, distress, worthlessness and loss (WHO, 2016). Generally, a number of older adults report feelings of isolation, loneliness and marginalisation (WHO, 2016). In the UK, in excess of 1 million older adults have not talked to relatives, neighbours or friends for at least a month (Age UK Buckinghamshire, 2016). However, in reality these numbers may be higher, as some older adults may not feel at ease with disclosing the ir circumstance to others (Tribe, 2017).

\subsection{Depression and Older People}

Across the lifespan, all people experience some type of an emotional or mental health need, and may require profess ional help with the ir mental health concerns (Tribe, 2017); However, specifically among older adults' depression has been identified as a mental 
health condition that is commonly experienced, affecting 10-15 per cent of this population (Baldwin, 2014; Huang \& Carpenter, 2011; Conner et al., 2010). Although depression may be commonly experienced later on in life, many older adults also report having good mental health and are living meaningful, rich lives (WHO, 2016). Furthermore, numerous older adults, having lived through and experienced difficult times, have successfully navigated through life; and in turn, demonstrate immense resilience (Centre for Policy on Ageing, 2014). Importantly, Resilience does not decrease with age; as a result, older adults are just as resilient as younger people (Gooding et. al., 2012).

Nevertheless, many studies have found that the older adult population are more vulnerable to mental health concerns. The reason being that older adults are more often than not exposed to significant life events such as: partner bereavement, retirement, the onset of a long-term health condition, social isolation, reduced functional ability and loneliness; as well as experiencing potential anxieties about poor health or death (Lane, 2017; Mental Health and Older People (MHOP), 2013). Similarly, Kamiya et al., (2013) suggests depression is prominent later on in life due to the impact of disadvantaged health in childhood, socio-economic challenges and pressurising life events. Therefore, given the evident concerns surrounding this population, mental health providers may need to consider strategies to help older people to maintain wellbe ing (preventative measures); and when required help older adult service users to access mental health services easily.

Kamiya et al., (2013) further argues that for some older adults' the reason these factors are prominent later on in life, links to the accumulative effect of a life time of difficulty showing itself through a number of health and economic discrepancies. In addition, depression experienced later on in life can be attributed to the collaboration of several factors throughout the life span - i.e. psycho social, genetic, psychological influences (Kamiya et al., 2013).In sum, older adults are particularly susceptible to the effects of depression (Conner et al.,. 2010), and unfortunately, is the primary reason of suicide among older adults (Mackenzie et al., 2006). Depression if left untreated can escalate the cost of health care, add to disability from other illnesses and shorten a person's life span (Baldwin et al., 2014). 


\subsection{Older Adults Mental Health Service Use}

Access to services should be determined by need and not age (Tribe, 2017); yet studies have identified numerous barriers to service use (including mental health) among the older adult population. Older adults have been shown as underrepresented on the caseloads of psychologists and counsellors (Goudie, 2003). When it comes to looking for help or treatment from mental health services, Conner et al., (2010) argues that older adults sought treatment less compared to any other adult age group. Research suggests older adults have a heightened awareness of stigma relating to mental health and that they are frequently considered by others to have negative attitudes to looking for help for mental health concerns (Mackenzie et al., 2006). What is more, the Improving to Access Psychological Therapies (IAPT), 2009 report on older people, found some older adults beliefs surrounding mental health problems as shameful, and that they believed it should be concealed from everyone - including health professionals.

Tribe, (2017) suggests older adults may have been conditioned to having a 'coping discourse' as many have lived through war and rationing; as a result, for those older adults' seeking help relating to feelings or having to display emotion, they might see this process as a sign of weakness or lack of 'moral fibre.' Other factors influencing the uptake of services among older adults may include resilience (as discussed earlier), discrimination, as well as recognising that many older adults' help seeking repertoire may not include accessing psychological and mental health services (Tribe 2017).

Although research in mental health services has shown barriers to service use among older adults, studies have also found noteworthy levels of engagement. Gum et al., (2006) found when given a choice, older adults preferred psychotherapy over pharmacotherapy. What is more, the study found the influencing factors underpinning their choice stemmed from previous experience with either treatment option. However, contrariwise, Watts et al. (2002) found when older adults received a referral to psychotherapy; these older adults' were likely not to attend the appointment or follow up. As a result, this could suggest that there may be significant barriers preventing older adults from initiating and/or engage in their preferred method of mental health treatment.

Interestingly, the idea of discussing feelings with a stranger is somewhat a new concept, within British society; likewise, the belief of the stiff upper lip often forms part of the main discourse for UK older adults (Tribe, 2017). As a result, this cultural phenomenon may decrease the number of people seeking medical or psychological help in 
comparison to countries with diss imilar cultural norms - including the cultural norms of older Black Caribbean adults living in the UK (Forbes et al, 2013). Nevertheless, therapy means a lot of things to different people, and contrary to some studies emphasising the negative help seeking attitudes of older adults, studies have also found older adults to have positive help seeking attitudes towards accessing services for mental health.

Mackenzie et al. (2006) study on older adults' conducted in Canada found the attitudes (among some older people) towards mental health service use as generally positive. Furthermore, the ir findings showed the older adults' in their study to be more motivated to seek help from primary care physicians compared to younger adults. Although, this study was conducted in Canada and holds a different public health service context(to the UK), it highlights the influence of a person's culture, beliefs and experiences on help seeking and accessing health services - including mental health. Consequently, as preference for seeking mental health service use appears to be led by the individuals' experience; exploring why Black Caribbean older adults who seem to want therapy but might not go may give further insight on culturally defined strategies to improve the ir engagement with services, as well as what influences this may have on service delivery and promotion of wellbeing and mental health services in the UK.

On the whole, despite the incongruity in research, it is estimated that approximately 50 per cent of people with depression (old or young) seeks professional help (Gulliver et al, 2012). Furthermore, research has found people regularly sourced alternative help (i.e. not from psychologists) for their mental health concerns from networks (i.e. relatives or friends), who did not offer scientific interventions' (Rickwood et al., 1994; Rickwood et al., 2007). Nevertheless, collectively, these studies give insight to some degree into the experiences of older adults accessing mental health services. However the methodologies used raises questions as to the richness and subjectivity of the data collected. Meaning, the reviewed studies have taken on a quantitative approach and although the data collected is helpful, the critical depth of these studies can also be enhanced by qualitative methods. Nevertheless, further research and reflection across disciplines is needed around the service use of older adults, as evidence has shown that mental health services are still not meeting the needs of older adults (Department of Health (DoH), 2014). 


\subsection{Ethnic Minority Older Adults' Accessing Mental Health Services}

Among the ethnic minority older population, research across the world has identified numerous barriers to mental health service use. However, in the UK, there are few studies on the Black and ethnic minority mental health use; as a result, the insufficient and inadequate amount of data on this population has been found to impact on the difficulty of treatments not being accessed, misdiagnoses and under diagnoses (Fundamental Facts about Mental Health (FFMH), 2016). What is more, in primary care, older adults from Black and ethnic minority groups have been found to have the highest risk of mental disorders not being detected (Borowsky et al., 2000). With all that said, the evident concerns surrounding mental health service use from Black and ethnic minority older adults, highlights the need for mental health professionals to further comprehend the decisions and experiences of service users that influence help seeking behaviour. In doing so, mental health providers may be able to deliver more effective, culturally appropriate and accessible services.

Reasons for the deficiency in service use among Black and ethnic minority older adults' may include: ageism, racism, different idioms of distress and help seeking (Tribe, 2017). Carr (2014) argues that people from Black and ethnic communities were reluctant to engage due to fear that services would be discriminatory, as well as trust being a primary and complex concern. Bowes and Wilkins on (2003) also noted previous poor experience of services as a main factor. Furthermore, Cinnirella and Leventhal (1999) argue the negative perception of psychological distress among Black and ethnic communities may result in the distress being hidden, only to be sought when problems become chronic or difficult for families to deal with.

It has also been argued that the low uptake of mental health services from older adults' may be due to people not ever having to access services previously. As a result, older adults with migratory histories may not know the role of the GP as a source of information and support for mental or emotional problems (Jacob et al., 1998), or they find it difficult to understand the services available and how to access them ( Bhui and Bhugra , 2002). The term 'mental literacy' has been used to understand why some older adults with migratory histories may not know how to access help for mental concerns (The Sainsbury Centre for Mental Health (SCMH), 2002). This term generally speaks of the absence of information surrounding mental illness rather than of services in mental health (SCMH, 2002). 
Nevertheless, whilst numerous studies have examined barriers to treatment amongst older people, there is a scarcity of qualitative studies that examine the lived experiences of Black Caribbean older adults - in particular Black Caribbean people and their perceptions when seeking help from wellbeing and mental health services. Furthermore, there are few studies examining how and what coping strategies are utilised by this populace (Conner et al. 2010). On the whole, the low uptake of these services by Black and ethnic older adults could potentially lead to their needs being unnoticed or underestimated by service commiss ioners (Fassil and Burnett, 2015). As a result, mental health professionals need to evaluate the pattern of service usage (among this group) to enable appropriate services being developed (Tribe, 2017). Furthermore, Black and ethic older adults' service users need to be involved in this evaluation (Morgan et. al, 2009), if not it could prove detrimental to the improvement of future mental health services for this population.

In the UK, Black and ethnic minority groups with mental illnesses appear to have different patterns of service utilisation (Bhugra et al., 2004 and Lawrence et al.2006). Bhugra et al., (2004) argued that the diverse pathways taken by some ethnic minority groups may be due to previous experiences with services (including mental health) and health beliefs. What is more, the diverse pathways may reflect help seeking attitudes towards services, which includes how culturally appropriate and appealing they are. Bhugra et al., (2004) further claimed once points of access are identified within this population, the weak points in pathways can be strengthened, therefore improving service provision and enabling access to services (Brown et al., 2010). Overall, the reported barriers to accessing services among the Black and ethnic minority population are said to be many; with available services being viewed as culturally insensitive, inadequate, inaccessible and inappropriate (Fernando, 2004; Fernando, 2014; Tribe 2014). In addition, MIND, (2013) argued that the National Institute for Health and Clinical Excellence (NICE) guiding principles may not always account explicitly or effectively for matters surrounding ethnicity or age.

Nonetheless, in multicultural environments, the needs of this increasing Black and ethnic minority population must be adhered to within mental health care systems (Fernando, 2014). It is crucial for action to be taken at every influencing level to ensure services are appropriate and accessible; as well as in line with anti-discriminatory practice (Tribe, 2017). Enabling a common/shared language between both service user and provider, improves service engagement by encouraging both to have an open 
dialogue, which could be understood by both. In sum, services for Black and ethnic minority older adults, need to be set up in ways which are appropriate and enables access which suits the requirements of this population; yet, this is not always the case (Tribe, 2017). Counselling Psychologists and other mental health professionals need to remain mindful of social contexts and discrimination in their work, as well as practice in ways that empower instead of control. By exploring the lived experience of Black Caribbean older adults' mental health service use, Counselling Psychologists and other mental professionals are in a position to better identify any weak points impacting on service use for our increasingly diverse UK population. Moreover, identification of these points may influence delivery and promotion of mental health services for this population.

\subsection{Help Seeking Theories}

There have been numerous philosophies and models applied to better understand help seeking for mental health difficulties; however these have not been broadly accepted (Gulliver et al. 2012). Although there is not a common framework, research exploring the levels and nature of help seeking has primarily examined three (wide-ranging) aspects of attitudes towards seeking help; these are: the motivation to seek help or beliefs; the objective for help seeking and the action of help seeking behaviour. Since there is evidence that attitudes and intentions can predict behaviour, the relevance of exploring attitudes to improve mental health service provision is important (Gulliver et al. 2012).

In addition, (as this discussed earlier in this chapter) stigma and discrimination are also considered as contributing factors to barriers for help seeking; similarly, they are often associated with mental illness (Corrigan, 2004). Further indicators may also include age and ethnicity, as older Black and ethnic minority adults do not appear to be seeking help from services (Royal College of Psychiatrists (RCP), 2009).

\subsection{Older Adults, Coping and Help Seeking}

There are five main reasons identified to impact on the well-being of older adults: physical-wellness; being poverty-stricken; being discriminated against; relationships and social support; and involvement in meaningful activities (Age concern and the Mental Health Foundation, 2006). However, the role of social support and its 
relationship to both older adults and mental health has been documented most considerably (Tribe, 2017). This means, within the older adult population, numerous studies have noted the significance of social networks (which includes close friends and family) as a mediating factor in improving mental health (in particular with depression) and wellbeing (Cornwell and Waite 2009). Furthermore, mental health professionals may also consider social prescribing as a way to mainta in wellbeing, and/or enable a better way for older adults' to access mental health services. Social prescribing strives to address an individual's need in a holistic way, as well as empower them to take control of their own health (The Kings Fund, 2017).

In addition, studies across the world have found religious faith as a substantial source of support and social capital among older adults' (Dein et al., 2019; Tribe, 2017; Kaplan and Berkman, 2016). For example, In the USA, Kaplan and Berkman (2016) found the largest form of social support for older adults outside their family was linked to religion and religious communities. Although, Kaplan and Berkman's (2016) report was based on an American population, some learning could be applied to the UK. Furthermore, Counselling Psychologists and other mental health professionals may also find it helpful to encourage UK older adults to talk about their faith and beliefs even if the mental health professional initially feels uncomfortable with it (Tribe, 2017).

\subsection{Black Caribbean Older adults, Coping and Help Seeking}

Across the world, research on help seeking among Black Caribbean people is limited (Woodward, et al. 2013). However, although studies are few; some studies in the UK have found that Black Caribbean people with mental health concerns tend to wait until cris is point before seeking professional help from services - including mental health (SCMH, 2002). As research in this area is limited, the doors of curiosity open to further explore and understand older Black Caribbean adults (and specifically), their perceptions, help seeking attitudes and coping strategies.

The type of coping an individual employs depends on various subjective components, which includes whether a person decides to use a particular strategy or not (Lazarus and Folkman, 1984). As a result, coping depends on an individual's understanding and judgment made about the situation/circumstance requiring a coping strategy (Lazarus \& Folkman, 1984). Goldburg and Huxley (1980) argue that patients' cultural evaluation of their difficulties and the interventions to help may differ from those of their GP's; therefore non-primary care paths become more appealing. For example, Lawrence et 
al., (2006) UK study found that Black Caribbean adults generally do not perceive consultations at the GP as a way to get help for depression; instead they turned towards 'getting on with things' as a method for managing emotional pain. Therefore, through gaining further understanding of these appraisals (within a cultural context) Counselling Psychologists and other mental professionals may develop ins ight into what this might look like for the psychologist and client pair - thus enhancing psychological tools to contain the therapeutic relationship.

Faith and religious practices such as prayer have been recognised as significant methods of coping among members of the UK's Black Caribbean community (Lawrence et al., 2006). As a result, religious, spiritual leaders or healers may be thought of as better suited and a sufficient base of help, rather than first-w orld models of therapeutic and/or psychiatric care. In addition, Woodward et al., (2013) notes the clergy are playing a key role in providing counselling for personal difficulties within the Black Caribbean community. However, contrary to previous studies, Woodward et al., (2013) also found significantly fewer Black Caribbean people (in their sample) sought council from a spiritual or religious provider. Furthermore, Woodward et al., (2013) study identified Black Caribbean people as less likely to be church members and less likely to take part in auxiliary congregation groups compared to African Americans and other ethnic groups (Woodward et al., 2013). The fact that the body of research found on Black Caribbean people stems from the USA highlights the limited research conducted in the UK. Although the USA holds a different context in regards to public health services, UK mental health professionals can still ga in some ins ight on coping strategies and help seeking behaviour among this population.

No matter what coping strategy is employed, this review of literature indicates the significance of subjective components that influence the type of strategy used for coping. As a result, by exploring the lived experiences of Black Caribbean older adults in the UK, the highlighted subjective components of their coping strategies may prospectively influence access, promotion and service delivery of mental services for this population. In addition, Black Caribbean older adults in the UK may also be found to not access services because they are doing just fine without them. Whatever the outcome, explorations of these experiences enable Counselling Psychologists and other mental health professional to engage with the subjectivity and inter-subjectivity, values and beliefs of participants. 


\subsection{Culture and Help Seeking}

Asnaani \& Hofmann, (2012) describes culture as a shared system of viewpoints, values and beliefs held by a specific ethnicity, race or geographical region. Every person holds a personal culture and all people are affected by aspects of the ir personal culture (Tribe, 2017). There are several components that inform our personal culture; these are (but not limited to): experiences of life family background, religion, ethnicity, individual and community values and meaning-making methods (Tribe, 2017). However, just because a person comes from a particular culture does not mean beliefs and views are shared. Holding this in mind may prevent stereotyping (Tribe, 2017). In addition, Lawrence et al. (2006) further suggests that the cultural disparity in these beliefs may cause inconsistencies in service provision.

A persons' cultural background may influence help seeking behaviour and how people label concerns relating to mental health and wellbeing (Tribe, 2017). The methods people use to preserve the ir psychological stability are partly establis hed and defined by cultural, societal and health rules; as well as the meanings which they assign to them (Fernando, 2014; Tribe 2014) Likewise, research has shown the construction of help seeking attitudes, mental health and illness (in terms of causation and treatment options or recovery) to be underpinned by a person's culture (Bhugra and Gupta 2011; Bhui 2013).

Harper, (2013) suggests the statistics held to describe the number of people having a mental health condition, to be heavily influenced by a person's culture. As a result, the statistics held are often challenged. In sum, age ing is culturally and soc ially constructed (Tribe, 2017). Therefore, to uphold anti-discriminatory practice, the experiences and views of older Black Caribbean adults will need to be openly discussed and explored. Furthermore, assumptions about how age ing is perceived by an individual or by his or her family or carers are also not to be made (Tribe, 2017). Upholding these practices not only improves mental health provision; it is also in line with aims of this study and with my values as a Counselling Psychologist.

All in all, several studies have illustrated the effect cultural ideas about mental illness have on attitudes and/or behaviours to seeking help in relation to mental health services (Furnham and Chan, 2004). However these studies have mostly taken a quantitative approach, and as mentioned earlier although the data collected is insightful, the next step is to expand qualitatively to continue to enhance the critical depth to of these studies. 


\subsection{Older Adults and Health professionals}

The needs of older adults are multifaceted. Therefore, mental health professionals will need to consider a holistic approach to enable older adults to sustain a good quality of life (McIntosh and Huq, 2017). Moving forward, by embracing a holistic approach, mental health service practice may be more helpful and anti-discriminative. This approach involves taking into account the older adults' personal history and inner resources (Tribe, 2017). In addition, with better resourced older adult services, this population can experience personalised support to sustain their sense of wellbeing. In turn, older adults may feel understood, respected and valued. Furthermore, the principles of anti-discriminatory practice can be upheld (McIntosh and Huq, 2017).

The RCP (2009) reported concerns that older adults were not getting the services needed and that policymakers did not deliver on improvements. As a result, the RCP (2009) launched a position statement on developing age appropriate, non-discriminatory mental health services. For these reasons, to assist with best practice for this population, mental health professionals and policy makers could consider thinking outside typical classifications and think more creatively. One idea is to consider how older adults may be segregated within their own older adult services (Tribe, 2017).

Fernando, (2014) argues that some health, social care and mental health professionals do not effectively consider within the service they provide issues of ageing, diversity and culture as a crucial part of practice. Bhui, (2013) also suggests staff may also lack understanding and training on the impact of both culture and mental health; or know how to provide culturally competent services. As a result, Black and ethnic minority older adults with mental health issues may not receive the most appropriate service, resulting in their needs being unmet. In addition, Black and ethnic minority older adults may experience serious discrepancies with mental health issues (Tribe, 2017).

Health professionals lack of familiarity with alternative health beliefs and idioms of distress may relate to Black and ethnic minority older adults' low levels of mental health service use (Shah, 2008). Nevertheless, it is important for every older adult's cultural and spiritual needs to receive active reflection, in order for improve service delivery (McIntosh and Huq, 2017). In sum, to not consider age and to treat everyone alike is discriminatory. Therefore, all health professionals will need to be trained to understand that Black and ethnic minority older adults may use different idioms of distress or explanatory health models (Patel et al., 2000; Tribe, 2017). 


\subsection{The Relational Preferences of Service Users}

Efficient mental health provision entails meaningful exchanges between service users and mental health providers and good/great communication (Wills and Holmes-Rovner, 2006). Health professionals understanding how Black and ethnic minority older adults differ in preference for relational style, is a crucial factor for improving services. What is more, this understanding can lessen inconsistencies in public health service delivery (Tucker et al., 2007). In addition, research has shown that mental health services who adapt their services to meet our diverse population needs to have better effective outcomes of care (Mulvaney et al., 2011).

A qualitative study conducted by Mulvaney et al., (2011) in the USA, among four ethnic groups reported good listening skills by health professionals as effective in coping and help seeking. What is more, this skill was specifically emphasised by African American service users in their study. The other skills highlighted as key for meetings with health professionals were: understanding, managing differences and spending time. In addition, Mulva ne et. al, (2011) argued that by health professionals comprehending and being open to the subtle yet essential differences in relational preferences, mental health services could improve levels of engagement among ethnic minority groups. As highlighted earlier, the USA public health context is different to the UK's; nevertheless, there is learning here, whereby psychologists and other mental health professionals can still gain some insight on how to reduce discrepancies in public health service delivery for a diverse population.

On the whole, a large number of studies employing quantitative methods have recognised a number of differences in preference for mental health service among ethnic groups; however, what seems to be absent from the literature are more in-depth qualitative research examinations (Mulvaney et al., 2011). With that said, although some qualitative research have investigated the distinctive relational attributes that are imperative in therapeutic relationships, their findings have not identified the differentiations in preferences for the delivery of care from health professionals across ethnic minority groups (Mulvaney et al., 2011).

To conclude chapter two, the current study hopes are to contribute to some gaps in the literature using a qualitative approach. By examining the experiences of Black Caribbean older adults, Counselling Psychologists and other mental health professionals could potentially plan collaboratively with service users to develop more effective, culturally appropriate and accessible services, to ensure the needs of this increasing 
populace are met. Furthermore, mental health professionals may better understand the ir perceptions, help seeking attitudes and coping strategies for mental health service use in the UK. 


\section{CHAPTER THREE: METHODOLOGY}

\subsection{Chapter Three Overview}

Chapter three examines in detail the rationale and philosophical underpinnings for the chosen qualitative methodological framework of Interpretative Phenomenological Analys is (IPA). In addition, the chapter includes a comprehensive account of how this study is conducted, which includes the collection of data and analysis.

\subsection{Rationale for Qualitative Research}

Qualitative research is considered best suited to understand individual differences, meanings and contexts (Yardley, 2000; Rennie, 2007). The reason be ing, qualitative research is designed to explore the idiosyncratic or subjective ways of an individual's experience (including how they speak and understand it), rather than, trying to measure the individual's experiences within a conventional framework which is already established (Willig, 2008). For these reasons, the qualitative framework is best suited for this study, as it a ims to understand better how older Black Caribbean adults in the UK make sense of coping with depression; as well as to understand better the decisions that older Black Caribbean adults make surrounding help seeking in relation to mental health services for depression.

Several studies have illustrated the effect cultural ideas about mental illness have on attitudes and/or behaviours to seeking help in relation to mental health services (Furnham and Chan, 2004); however, these studies have mostly taken a quantitative approach and although the data collected is insightful, the next step is to expand qua litatively (in line with the work of Lawrence et al., 2006) to continue to enhance the critical depth of these studies.

In addition, while a number of studies have examined barriers to treatment amongst older adults, there is a scarcity of qualitative studies that examine the experiences of Black Caribbean older adults and their perceptions of barriers faced when looking for help from wellbeing and mental health services. As a result, conducting qualitative research may highlight issues that affect seeking help in relation to mental health services for depression; as well as the coping strategies used among the older Black Caribbean population in the UK. Furthermore, factors found may potentially aid 
counselling psychologists and other mental health professionals in developing appropriate and accessible services. These strategies of engagement can be defined culturally to meet the needs of this growing population (Bhugra and Gupta 2011; Bhui 2013; Fernando, 2014).

The fact that many of the studies reviewed have taken a quantitative approach suggests that this is an important topic as it appears to bring to light the concerns about ethnic minority older adults' use of mental health services. Nonetheless, it is important to note that a considerable amount of quantitative research are underpinned by positivist ideas; which in turn, may result in the subjective meaning for an individual appearing absent from their findings. Subjective meaning is essential to expanding mental health professionals' knowledge and understanding of the possible realities experienced by service users, and specifically in relation to this study, how older Black Caribbean adults make sense of coping with depression.

\subsection{Rationale for Research Paradigm}

A research paradigm sets the context and theoretical positioning of a researcher's study. Research practices and methods are guided by paradigms; that is, paradigms reflect an individual's perception and understanding of his or her's social environment. Each paradigm contains various diverse views around ontology (the origins of 'truth'/ realism), epistemology ( the creation of knowledge), axiology ( the principles of the investigation), rhetoric (the part partic ipants and researcher play in investigation) and methodology ( systematic accuracy) (Hays \& Wood, 2011)

Kasket, (2012) notes that the Counselling Psychologist researcher is open to exploring many of the paradoxes, divergences, perspectives and research approaches available. This may be influenced by the core principles of Counselling Psychology, which considers a holistic approach when working with individuals' (Karademas, 2009); As a result, it can be argued that the Counselling Psychologist researcher may hold a similar perspective when undertaking research.

Nevertheless, there are many paradigms used to guide research (Ponterotto, 2005). Furthermore, different approaches to research can be very profound with varieties that may be incompatible with each other (Willig, 2008); all in all, there is no conventional way amongst scholars on how to classify these epistemological frameworks (Avenier and Thomas, 2015). As a result, a traditional categorisation relies on a dualistic partition 
between positivism and anti-positivism or positivism and interpretivism (Avenier and Thomas, 2015).

Brief descriptions of three paradigms often discussed in texts for Counselling Psychology research follow:

\section{3a Positivism Paradigms}

Positivism sits within a realist ontology (Mertens, 2005). Through the lens of a positivist, an individual's experience or a theory is examined or tested by measurement or observation (O'Leary, 2004). As a result, primarily, quantitative methods are underpinned by this paradigm, though qualitative methods can also be used (Mertens, 2005).

\section{3b Post-Positivism Paradigms}

Post-positivism sits within a critical realist ontology meaning there is an objective reality one is determined to understand (Mertens, 2005). Through the lens of a postpositivists the world can be ambiguous, ever changing and hold a number of realities (O'Leary, (2004). For these reasons, post-positivism can be seen as both holistic and intuitive, as well as both investigative and inductive (O'Leary, 2004). Qualitative methods are primarily used by post-positivists, though quantitative approaches can be applied also (Mertens, 2005).

\section{3c Interpretivist-Constructivist Paradigm}

Interpretivism-constructivism sits within a relativist ontology which distinguishes many realities, values subjectivity and hypothesises which argue meanings are co-constructed between researchers and participants (Ponterotto, 2005). Researchers who are interpretivist - constructivist typically have a tendency to depend on participants interpretations of the situation being examined and considers the influence of the researcher's personal experiences and background on the research process (Creswell, 2013). As a result, through the lens of this paradigm, reality can be considered as socially constructed (Mertens, 2005). In this paradigm qualitative methods as well as mixed approaches are used (qualitative and quantitative). 


\subsection{Rationale for a Critical Realist Position}

After review of the common paradigms in Counselling Psychology research, this study draws from an Interpretivism-constructivism world view and embraces an episte mological position of critical realism - positioned between the opposing camps of (naïve) realism and (radical) re lativism. This centre position allows for the authenticity of experiences to be valued, while not without rooting itse lf in positivism's naive realism, social constructionism or relativism camps (Bentall, 1999).

As discussed earlier, researchers can take a range of different positions and styles within an adopted approach (Miller, et al., 1997; Kasket 2012). As a researcher, I hold the view that ( independent of an individual's understanding), attitudes, feelings and physical conditions exist ; however due to a person's expectations, values and subjectivity they may be unable to easily and openly access them (Madill et al., 2000). For these reasons, I als o consider social constructionist thoughts; meaning, I believe that the construction of knowledge of the world as well as 'shared understandings' occur amongst people. Furthermore, critical realism is also compatible with this concept. Therefore, in line with the aims of this study and while adopting a critical realist perspective the author is assuming that depression exists and that exploration of help seeking views among older Black Caribbean adults in the UK can be useful for developing appropriate culturally defined pathways for help seeking to meet the needs of this population.

\subsection{Rationale for Interpretative Pheno menological Analysis (IPA)}

IPA aligns with the aims of this study, the epistemological stance taken and Counselling Psychology values. As discussed in chapter one, Counselling Psychologists embraces a person's subjective or inter-subjective experiences and adheres to humanistic phenomenological principles. As a result, the values of Counselling Psychology is not only consistent with this study's aims (to curiously investigate and contextualise the UK's older Black Caribbean adults' help seeking views and experiences of coping with depression), the methodological tool (IPA) proposed to analyses the data collected is congruent too as it thoroughly explores how participants understand the ir own realities . For these reasons, IPA is considered IPA best fit.

IPA strives to give participants both a voice whilst comprehending the ir experiences and concerns (Larkin et al., 2006). This sense of balance is referred to as the 
hermeneutics of empathy (which is the phenomenological component of examination) versus the hermeneutics of suspicion (the interpretative component of examination) (Larkin et al. 2006). By following this method, it enables the exploration of older Black Caribbean experiences, meanings to be carefully thought about, and a deep enquiry of the data. With that said, the researcher remains mindful about the considerable variability in how other researchers use definitions and how they work in practice (Willig and Stainton-Rogers, 2008).

In addition, Reid et al., (2005) argues subjects that are underexplored are better matched with IPA. Help seeking among Black Caribbean people is limited (Woodward, et al., 2013); furthermore, while a number of studies have examined barriers to treatment amongst older adults, there is a scarcity of qua litative studies (Conner et al., 2010). For these reasons, IPA was also considered for this study. Although IPA is considered best suited for this study, the researcher acknowledges that the analysis also has its limitations. For example, the role of language in IPA is critic ised. The reason being, it has been argued that language creates rather than describes reality- meaning when a person describes an event, they are in fact creating a version of that experience (Willig, 2008). However when adopting an IPA approach, participants described their experiences to the researcher through the language they use; as a result, this may be seen as compromising the accuracy of participants' accounts.

In addition, it has also been argued that although IPA can describe the lived experience of an individual, it does not go further than to explain it (Willig, 2008). However, it is argued that before the researcher can explain an experience, it must first to be understood as well as the meanings people attach to the ir experience explored (Macran and Shapiro, 1998). Nevertheless, IPA synthesises concepts from phenomenology and hermeneutics. This results in a method which is descriptive as it is concerned with how conditions emerge and letting these situations speak for themselves, and interpretative as it distinguishes that there is no such thing as an uninterrupted phenomenon (Pietkiewicz, Smith, 2014).

All in all, the researcher in this study recognises that similarly to working with service users or clients' problems, there are several approaches to examining and conducting research (Kasket, 2012). Therefore, when engaging in research, a pluralistic attitude is warranted; this attitude involves the recognition of various research methodologies (Kasket, 2012). For these reasons, being a Counselling Psychologist researcher does not equate to being either a qualitative researcher or an Interpretative Phenomenological 
Analys is (IPA) researcher (Kasket, 2012). Researchers' methods and paradigm positioning can differ within an assumed approach; therefore, when thinking about this study, the researcher will remain curious about the whole host of research approaches available, as well as considering first the question-the object of inquiry and then openly considering the ways in which this may be explored (Kasket 2012).

\subsection{Explanation of the Study's Design}

Eight interviews were conducted with four men and four women aged between 65 and 79 years old. The interviews were semi-structured and conducted on a one to one. All identified as Black Caribbean in origin but living permanently in the UK. Interviews lasted between 30 and 120 minutes. The Interviews were conducted by the researcher, audio-recorded, transcribed verbatim and analysed.

\subsubsection{Data Collection}

Interviews are frequently used as a means of collecting data in qualitative research, as the data generated from this method can be analysed in a variety of ways; moreover, there are usually fewer logistical obstacles to overcome in arranging them (Willig,2008).

\subsubsection{Construction of Interview Schedule}

Interviews that are semi-structured allows for particular research questions to be answered as the direction of questioning remains under the control of the researcher (Creswell, 2003), enabling participants to contribute fresh ideas as well as the ir own insights (Willig, 2008). In addition, initial questions on semi-structured interviews can be adapted where necessary to enable further probing of interesting discoveries which may come up (Smith et al., 2009). Furthermore, this approach gives an added advantage to the Counselling Psychologist researcher as it enables acceptance, understanding and authenticity in establishing relationships and rapport with the participants in the study (McLeod, 2003).

Salient topics which emerged from literature in this area helped to construct the semistructured interview schedule. Topics in this area were expanded on using open ended questions, with guidance to make sure that certain questions did not lead the participants (Smith et al., 2009). Interview questions were used as a guide only and not in a 
prescriptive style (see appendix A). In addition, the researcher conducted a pilot interview to determine clarity and structure and clarity of questions asked.

\subsection{Criteria for the Inclusion and Exclusion of Participants}

The criteria for participant's participation were:

- Male or female aged 65 years old and over

- Identifies as of Black Caribbean origin

- $\quad$ Lives in the United Kingdom

- Personal experience of self-defined depression both now and/or when younger

This study is concerned with understanding older Black Caribbean adults' personal experiences, context and meanings that underlie help seeking views and behaviour in relation to self-defined depression. Therefore, participants recruited were required to have some personal experience of self-defined depression across their life span. Participants were not required to be clinically depressed or have a formal diagnos is as the researcher's primary concern was with the views of the general public and/or potential service users. The reason being, the researcher wanted to limit where possible bias views towards accessing help for depression from mental health services by participants who are actively involved with these services.

In addition, the term self-defined depression is used to enable each participant's unique subjective views about depression and help seeking for depression to be heard and understood. Interview questions foc used on partic ipants, unique/personal understanding of depression and how this understanding impacted on participants' personal experience of coping with depression. Furthermore, to help filter some potential biases due to active involvement with mental health services, recruitment from primary care or any mental health services was avoided; moreover, service users who are prescribed medication from the ir GP/or secondary care, to cope with low mood or have an active referral to psychiatric care were excluded.

\subsubsection{Recruitment Procedure}

An advert/information sheet was sent via email, as well as telephone contact to key members and organisations within the older Black Caribbean community (see Appendix 
$\mathrm{B}$ and $\mathrm{Ba}$ ). Key members identified several potential participants, who met the research criteria and sent them the information sheet. Potential participants responded with interest and provided their telephone contact information to be forwarded on to me. I contacted each participant to discuss /clarify any further details of the research, as well as to conduct a brief screening process to confirm that the inclusion and exclusion criteria were correctly met. In addition, confidentiality and consent to part icipate was verbally explained during this contact. Once verbal consent was given by participant, we arranged a meeting at a convenient time and date. No potential participant refused to participate. Interviews took place, in a private setting of participants' choice where both participant and researcher felt safe to proceed (i.e. a private room in an organisation's building).

Initially, the study proposed to recruit participants from day centres and lunch clubs only, however all participants were recruited through the "Snowballing" method, meaning participants passed on information about the study amongst their social networks (relatives or friends), which proved to be effective. Interestingly, the snowballing method has been found to be a valuable approach for when the subject matter under study is stigmatising or for the members of society who are considered as "hard-to-reach" (Magnani et al., 2005; Sadler et al., 2010), demonstrating its appropriateness for this study.

\subsubsection{Sampling Criteria}

When considering the sampling criteria, the researcher must ensure selection is theoretically congruent with the study's paradigm and framework for analys is. As a result, the research sample was considered purposively instead of through probability approaches (Smith et al., 2009; Chapman and Smith, 2002). Also, when considering participants, the selection must enable the researcher to access views on the subject under investigation; they symbolise a point of view, rather than people on the whole. What's more, as IPA intends to comprehend a specific context or phenomena (an idiographic method), small groups sizes are acceptable when conducting research (Smith et al., 2009; Chapman and Smith, 2002). 


\subsubsection{The Sample}

Eight participants were recruited for this study. Eight to twelve interviews has been suggested as needed for a sufficient data analysis, as well as meet measures for data saturation (Guest et al., 2006).

\section{Table One}

Depiction of the selected s ample for this study

(Table 1) presents a summary of the selected sample and demographic information.

\begin{tabular}{cccccc} 
Pse udonym & Gender & Age & Ethnic origin & $\begin{array}{c}\text { Aged arrived to the } \\
\text { UK }\end{array}$ & Marital Status \\
\hline Earl & Male & $73 \mathrm{yrs}$ & Black Caribbean & $19 \mathrm{yrs}$ & Single \\
Mary & Female & $69 \mathrm{yrs}$ & Black Caribbean & $17 \mathrm{yrs}$ & Widowed \\
Freddie & Male & $65 \mathrm{yrs}$ & Black Caribbean & $20 \mathrm{yrs}$ & Married \\
Shirley & Female & $69 \mathrm{yrs}$ & Black Caribbean & $15 \mathrm{yrs}$ & Married \\
Vincent & Male & $79 \mathrm{yrs}$ & Black Caribbean & $19 \mathrm{yrs}$ & Divorced \\
Beverley & Female & $69 \mathrm{yrs}$ & Black Caribbean & $17 \mathrm{yrs}$ & Married \\
Leroy & Male & $74 \mathrm{yrs}$ & Black Caribbean & $18 \mathrm{yrs}$ & Married \\
Margaret & Female & $73 \mathrm{yrs}$ & Black Caribbean & $19 \mathrm{yrs}$ & Married
\end{tabular}

\subsection{Interview Procedure}

The researcher informed the participants that the interviews will be face to face, audiorecorded and semi-structured. They were further informed of the study's confidentiality policy. Each participant was given a consent form and a further detailed information sheet about the nature of the study and their involvement (See appendix B and C).

Once interviews were completed, participants were debriefed (see appendix D). This debrief sheet included sign-posting information for relevant services, where needed, if any feelings of distress occurred or if participants wanted to further explore things they talked about at the interview. Subsequent to interviews, the researcher reflected on the ir own thoughts and feelings, and how they may have impacted on the process, noting them down. To conc lude, interviews were transcribed (verbatim) and examination of the 
data followed.

\subsection{Data Examination}

The examination of data followed the IPA procedure as explained by Smith et.al, (2009). Although the IPA method has specific protocols, it is not prescriptive; as a result there is no precise way to perform it. Nevertheless, with the guidance of research supervision (by an experienced psychologist and researcher, with a wealth of know ledge on issues relating to this study), the analytic process was supported. Having this support encourages quality qualitative research, alongside guiding papers (Yardley, 2008).

\subsubsection{Steps of Examination}

IPA data analys is entails a range of steps. Step one, involves listening to the audio of the interviews whilst reading and re-reading the transcripts. In doing so the researcher familiarises themselves with partic ipants narratives (Smith et al., 2009). At this point, first thoughts are recorded and are referred to as free textual analys is (Smith et al., 2009). In addition, noted on the transcript are tentative exploratory comments, made to capture anything significant or fascinating pertaining to participants in relation to this study's objectives. The researcher aimed to keep initial notes close to the participant's individual viewpoint.

The second step of analysis identifies the label or themes that characterise each section of the text. In this stage the analysis shifts from the main transcript to focusing on the initial notes. These initial notes are explored and developed into emergent themes. The researcher's focus here is to develop emergent themes that capture an understanding of participants' experiences, thoughts and emotions. This requires the researcher to construct a succinct phrase which highlights initial notes attached to the transcript. At this stage, the 'I' in IPA becomes more alive as there is more of an interpretative focus.

The third step attempts to establish structure within data analys is. The highlighted themes in step two are organised and evaluated. Themes echoing parallel ideas are grouped together; forming clusters which in turn enable the creation of superordinate themes (Smith et. al., 2009). The researcher also verifies the superordinate themes through returning to the transcript to evaluate; this is to ensure superior ranked themes best reflect participants' experiences captured. 
In addition, at this stage the researcher attempts to bracket ideas from preceding interviews by breaking from analysis, and in turn this allows for clearer thinking when attempting to examine the next interview. Though similar ideas were acknowledged, the new ideas that emerged were prioritised; as a result, the researcher then notes the parallels and dissimilarities' amongst participant's accounts (Smith et al., 2009)

In step four of examination, connections across participants' transcripts are closely examined. Superordinate themes from each transcript are listed and emergent themes repeated amongst transcripts are promoted to the subordinate level to enable crossanalysis. Following this, themes are re-structured and summarised in a table. In the final stage of analysis, the concluding themes will move to reporting with statements that explains the researcher's interpretation characterised to the accounts of participant's (Smith et al., 2009).

\subsection{IPA's Quality and Accuracy Process}

The pursuit of quality investigative studies is based on the common concepts and principles such as reliability, inferences quality, constructs quality and generalisation. These concepts/principles are essential in any research if it intends to be valued as being rigorously conducted (Avenier and Thomas, 2015). In addition, the specific habit of constructing quality data collection and analysis depends on a study's epistemological framework (Avenier and Thomas, 2015).

Yardley, (2008) suggests when considering the validity of a study, that the researcher assesses how their study is conducted and whether the study results are rigorous, valuable and trustworthy. Interestingly, if the researcher evaluates their research holding quantitative philosophies' the results become unsuitable as quantitative research epistemological bases are generally different (Yardley, 2008).

A number of the literature reviewed for this study failed to be explicit with their epistemological frameworks; however, Lawrence et al., (2006) study did this. In this study, the authors explored the beliefs that underlie help seeking behaviour within older adults from three ethnic minority groups; what's more this particular study inspired the researcher to undertake this current study; however the ir data analys is was based on the Grounded Theory approach.

Grounded Theory is a qualitative approach often compared with IPA. Charmaz (2006) suggests that Grounded Theory grew from sociological roots. This approach is 
intimately allied to positivist and post-positivist assumptions, meaning by remaining objective, knowledge may be obtained and the focal point is to create an extensive hypothesis. The positivist and post-positivist assumptions stance diverges from IPA; the reason being, IPA embraces hermeneutic-phenomenology as its foundations, and connects better with the interpretivist-constructivist paradigm instead of the positivist or the post-positivist paradigms (which Grounded The ory also adheres to). For these reasons, this study rejected the Grounded Theory approach as the current study's aims and epistemological stance is not congruent with Grounded Theory.

The guidelines employed to assess the validity and quality of this study derives from Yardley (2008). Yardley (2008) suggests that in research, there are four core principles for evaluating quality; these principles are also advised by Smith et al., (2009). These principles are: Sensitivity to context, Commitment and Rigor, Coherence and Transparency and Impact and Importance. Descriptions of the principles follow below.

\subsection{0a Sensitivity to Context}

The researcher (in relation to this principle) considers the relevant theoretical and empirical literature underpinning the ir study. The aim here is to identify gaps in an individual's psychological understanding of a topic resulting in the facilitation and development of appropriate research questions (Yardley, 2008). In addition, IPA illustrates sensitivity to the participants' data; meaning, that the study can evidence the ir findings (interpretations and themes) through the considerable amount of quotations acquire d (Yardley, 2008; Smith et al, 2009). As a result, participants' are given a voice through this process. To conclude this principle, the researcher must relate their discoveries to applicable literature within their discussion chapter.

\subsection{0b Commitment and Rigour}

In this principle, in order for the researcher to extract meaningful and rich data, the data collected is analysed extensively with adequate depth. Yardley (2008) notes that a researcher's best hope is that during thorough micro-analys is of participants' accounts, about how the participants experienced the topic/ study at hand, insightful findings will be produced. In this study, the knowledge and skills needed to proceed with this process was gained through the methodological lectures on the professional doctorate in Counselling Psychology training. Furthermore, the researcher thoroughly immersed themselves in the literature relating to the research topic. 
In addition, in order to remain in line with the principles of IPA, the researcher ensured the sample was homogenous by screening participants against the inclusion criteria prior to interviewing (Smith et al., 2009). Furthermore, while interviewing, the researcher probed participants (where necessary) to stimulate more interesting and indepth findings. All in all, the researcher thoroughly engaged with the analys is process; and the rigorous coding process (Smith et al., 2009), which enabled the capturing of participants realities and/or experiences to reflect in this study.

\subsection{0c Coherence and Transpare ncy}

This principle examines the study's investigating questions, paradigms and the approaches used for analysis. In doing this, the researcher presents a firm argument for their study as well as firm justification for how it has been conducted; this in turn optimises the coherency of their study. Furthermore, to attain transparency the researcher provides a comprehensive account of the investigating process and approaches performed; as a result, this helps readers to better understand the protocols involved in their study.

\subsection{0d Impact and Importance}

In this principle, research is considered valid if something important, fascinating and valuable is evidenced (Yardley, 2008; Smith et al., 2009). For these reasons, the researcher uses the introduction and literature review to highlight the necessity for the ir study.

\subsection{Ethical Considerations}

Ethical approval was obtained for this study, from the university where the researcher attends (see Appendix E). The study did not require NHS ethical approval due to the nature of this study.

The nature of the work with older adults can highlight a number of issues where trainees or clinicians may need to make sense of, and seek support with, when required (McIntosh and Huq, 2015). The researcher, who reflects on one's own practice, engages in professional and personal consideration, and analys is that require the researcher to think about how the ir interaction with the ir participants impacts on them, as well as how the participant may have experienced that encounter (McIntosh and Huq, 2015). 
For the reasons detailed above, potential ethical dilemmas for this study was reflected upon and addressed in supervision. Moreover, through supervision the researcher's skills as a therapist was considered potentially helpful when managing the possible distress of participants and potentially providing initial support.

Participants were given a letter detailing the purpose of study; and a consent form was given and explained to each participant (see appendix B \& C). Importantly, all participants willingly took part in this study; as well as there being no withdrawal requests from the study. Furthermore, the researcher also provided each participant with information for further support should they require it (see appendix D) following the ir participation in the interviews.

In addition, the researcher made follow-up calls to each participant after interview to ensure that they were not negatively affected by the interview. All the participants reported not needing further support and that they felt fine following the interview. Transcripts (digita1/hard copies) and recordings were stored securely on the researcher's password protected laptop at the researcher's home in a key locked cabinet. Participants' anonymity was assured by using pseudonyms and eradicating all identifiable information. 


\section{CHAPTER FOUR: RESULTS}

\subsection{Chapter Four Overview}

Chapter four illustrates the results extracted from the systematic examination (Interpretative Phenomenological Analys is (IPA)) of eight Black Caribbean older adults 'personal experiences, help seeking views and perceptions of coping with depression. Three master themes emerged through the analysis:

1. "If you don't know, you don't seek help"

2. “...I was depressed...I knew I was depressed"

3. "You have to decide": Attitudes to Help Seeking and Mental Health Service Use

As discussed in Chapter three, the researcher developed the themes above, by keeping close to the double-hermeneutic principle of IPA; this means the researcher made a considerable effort to make sense of the participant, who is also at the same time making sense of their experiences and views of coping with depression. Importantly, the themes developed do not cover every facet of Black Caribbean older adults lived experiences and views of coping with depression. Nevertheless, these themes were chosen due to their value to the participants' in this study, as well as their relevance to the specific investigatory questions asked of this study.

Terms that are descriptive (for example 'all' 'some' and 'others') are used to signify the general collective points of participants views. In addition, in order to respect and protect the participant's identities, pseudonyms have been applied to each one in this study. Furthermore, any information which could possibly be identifiable and/ or personal to participants has been modified or deleted. To improve readability of interview excerpts, minor modifications were made to its presentation. Hesitations (e.g. 'errm') and repetitions or filler words (i.e. 'like') have also been deleted from excerpts to enable further clarity for the reader. 
(Table 2) presents a summary of the master themes and subordinate themes.

\begin{tabular}{|c|c|}
\hline Master Themes & Subordinate Themes \\
\hline \multirow[t]{4}{*}{$\begin{array}{l}\text { 1. "If you don't know, you don't seek } \\
\text { help" }\end{array}$} & $\begin{array}{l}\text { Participants' Experiences of Migration } \\
\text { in relation to Depression }\end{array}$ \\
\hline & "Just get on with it" \\
\hline & $\begin{array}{l}\text { Participants' Significant Relationships } \\
\text { Experiences in relation to Depression }\end{array}$ \\
\hline & $\begin{array}{l}\text { Participants' Experiences of Health } \\
\text { Professionals and Listening Skills }\end{array}$ \\
\hline \multirow[t]{2}{*}{$\begin{array}{l}2 . \text { “....I was depres sed...I knew I was } \\
\text { depressed" }\end{array}$} & Grief in Later Life and Depression \\
\hline & A matter of "Inconvenience" \\
\hline \multirow{4}{*}{$\begin{array}{l}\text { 3. "You have to de cide" : Attitudes to } \\
\text { Help Se eking and Mental He alth } \\
\text { Service Use }\end{array}$} & "...Recognise and Start ..." \\
\hline & $\begin{array}{l}\text { "Why should I go tell somebody my } \\
\text { business?": Challenges and cultural } \\
\text { beliefs }\end{array}$ \\
\hline & "Just get on with it" part 2 \\
\hline & "No guarantee": Professional services \\
\hline
\end{tabular}




\subsection{Overview of master the me one: "If you don't know, you don't seek help"}

Master theme one sets the scene for the following themes. This theme reveals how in earlier life participants made sense of coping with their self-defined depression and what influence this experience had on their help seeking views. All partic ipants report experiencing their self-defined depression pre-age 65 years; however, the context in which each participant's depression occurred varied. In addition, all participants discussed challenges to seeking help for their depression at the time.

Participants' experience with depression pre-age 65 years is summarised within four subordinate themes:

- Participants' experiences of migration in relation to depression

- "Just get on with it",

- Participants' significant relationships experiences in relation to depression and,

- Participants' experiences of health professionals and listening skills.

\subsubsection{Participants' Experiences of Migration in relation to Depression}

This subordinate theme reflects the influences migrating to the UK had on participants' self-defined experience of depression and how this subsequently impacted on participant's help seeking views in relation to depression. Participants described a process of discovery, which involved learning to adapt to a new country, where by the lack of knowledge about depression, mental health illness and services presented as a challenge to help seeking at the time.

In the excerpt below, Mary depicts her self-defined depression. Her story illustrates how she entered a new country where she did not have formal knowledge about her selfdefined depression, and how this inhibited her ability to seek help at the time.

"When I first came in this country... I was depressed (gasped) but I didn't know it because in those days we didn't hear the word depression... didn't know what it was, didn't hear the word stress or depression so I didn't know I was depressed... I had left home, sunny sunny climes, all my friends... I left my comfort zone as it were... and I came to this country...in those days there was a lot of smog... everywhere was grey... it was in the month of September... I remember just crying, every letter I wrote to friends... were blotted with tears... I was just a very sad 
person but I didn't know I was depressed at the time because it wasn't a word that was used..."

Mary further elaborates:

“...the thing is ... not knowing that I was depressed ... if you don't know you don't seek help.... I just know that I was very unhappy... I couldn't ask for help because there wasn't labelling in those days.... it's in later life ... long after I had my children... when it really registered that there was a problem...I went into higher education and I had to study psychology, then I realised that, it was a problem... that it was depression.. yeah..."

Shirley echoes some of Mary's experience:

"...when I was 15 and came to England ... that was a very depressing time.... and why was it depressing... because I cried for 3 months. I remember we came in September.... left a happy life, my friends, my familiarity, and everything that I knew of, to come to a very... at that time I felt it a very depressing country you know... cause it was very dark, very dull, very dismal ... maybe I felt hopeless now I'm thinking of it... maybe I thought it was a hopeless situation... but that was my first as far as I can think back ... because I don't think that I was ever depress before, I didn't even realise I was depressed then to be quite honest with you so I would say my first experience was coming to England in 1962 at the age of 15."

In these excerpts, Mary and Shirley reflect on their understanding of depression, and connect the ir understanding with the ir experience of migrating to the UK. "I didn't know" , "didn't realise or "I don't know", is a phrase reiterated in their accounts, which suggests given the depth of their experience that there was an awareness that mood and behaviour had changed but the discourse wasn't there to name it. In addition, participants' self-defined depression depicted here appears to be reactive (followed either a stressful or traumatic event) rather than endogenous (biological influences).

Being able to "label" as exemplified by Mary or name their self-defined depression seems key to seeking help at the time. Below, Shirley further shows how her experience of migrating, lack of knowledge about depression, mental health illness and services influenced her help seeking views and seemingly the attitudes of others within the Black Caribbean community.

"I wouldn't have known because I mean in those days in the 60's everybody come here and they come here to work and that's all... you come to work or come to study and that's everybody's mindset...you work or your studying to come back home... so you weren't looking ...I don't know if there was help available but you weren't looking for any help because you were just looking between you and your local environment...my uncle knew I was not happy but what could he do but say girl just behave yourself...stop your nonsense type of thing ..." 
She further elaborates:

“... there was no time to pamper you with your problems ...because I've far as they're concerned you should be lucky ... you should be lucky that at least you are somewhere where there's opportunity and the opportunity they're talking about is finding yourself a job, you know that you couldn't find back home or educating yourself.... so even if there was somewhere to go for help ... we didn'tknow about it because we just kept in our little community."

Finally Shirley states:

"...the English people were not friendly and they were not accommodating... so you're not going to go to them for help...."

Like Shirley, Vincent describes a similar mindset within the Black Community at the time:

".... a lot of us, we come from the Caribbean, if some of us were lucky... we get a room... we go to work, we come home; you go to work, you come home..."

To conclude, Shirley and Vincent's excerpts illustrates how at that time, the "opportunity" to come to the UK to better one's se lf with "work" was crucial within the Black Caribbean community. This key focus appeared to over-ride desires to seek help specifically for their self-defined depression, as Shirley hints "no time to pamper you with your problems". Moreover, it seems while individuals within the community were learning to adapt to a new way of life in the UK, people were also "not looking for help" outside this focus, regardless of the impact on their mental health following the ir migration. Also social exclusion and racis $m$ are alluded to and the difficulty of being seen as part of the wider community. As a result, these aspects expressed by participants appeared to have challenged help seeking at the time for their depression, as well as strongly influencing participants help seeking views in relation to depression.

\subsection{2 "Just get on with it"}

This subordinate theme demonstrates the influence a self-sufficient outlook had on participants' help seeking views when experiencing their self-defined depression. Participants' accounts gave a sense to "just get on with it" as if there was no other choice but to deal with ones experiences on their own. Margaret's excerpt below further illustrates this: 
"...I was unhappy; I was disappointed - many, many times disappointment comes into my life in different ways... But I never really thought that I was depressed... maybe I was but, you know, Ijust thought, well, I had to get on with life. I had to do what I had to do and that's what I did..."

She further explains:

"...I know people say they go and they get counselling but I didn't... well, I think the reason why I was depressed, I didn't think that I needed to go and talk to anyone about it. I just felt like I could cope with it as I said."

Lastly Margaret states:

"I struggled along with the pregnancy, I had my baby and I just got on with it."

Interestingly, to "just get on with it" is a mindset also highlighted within the participants' experience of migration' in relation to depression theme accounts; indicating, perhaps this is an embedded cultural norm of the time. What is more, among participants, to "just get on with it" also seemed to be motivated by a sense of being "depended on" by their family; suggesting also strong influences of cultural or family values on help seeking attitudes at the time. Beverley depicts this further in her excerpt below:

".... for me, my post-natal, as a mum, I had to do it. I had to do the work in the house even though I couldn't cope. So I didn't tell much people that and I didn't care if they wanna come and see the house dirty; that's their business..."

Margaret echo's:

"I was never down, that low that I couldn't function because as far as I'm concerned, those five people depended on me and I was determined to be there for them come what may."

Vincent concludes:

"I think ... what really helped me along is this working... steady working because my idea is this...my wife and my children, it's up to me to go out there and work for them..."

The need to provide or care for one's family independently seemed to be key to coping well with participants' self-defined depression. This key focus also appears similar to the experiences depicted in the 'participants' experience of migration in relation to depression' theme; as here too participant's drive als o seems to over-ride desires to seek help specifically for their self-defined depression. 
Other participants' accounts gave a sense of personal development, distraction, strength and resilience as a way of coping with the ir self-defined depression at the time. Freddie excerpt below further illustrates this:

"...you asked how I got out of that so I suppose you start to spend more time identifying with those things that strengthens you rather than those things that actually weakens you....so my mind was always on what I loved at that time reading, so I could escape and read a book..."

He further elaborates:

"I was trying desperately not to damage anybody so the getting out of the environment for that period was more or less beneficial for me now that I look back at it."

Leroy depicts a similar experience.

"If there was any help it was the fact that you can escape and when you had to go to a certain place to do a certain duty... you might hang about longer in that area than you probably should..."

Freddie and Leroy highlight a need to psychologically escape from the ir self-defined depression, to source help from within instead of sourcing out external help. Below, Shirley also speaks of another form of escapism through music:

“...I've learnt that music really lifts depression...lifts you when you're feeling low... and then when becoming a Christian I realised even when David was down he used worship music to lift his soul... so for me I realised that music really keeps me when I'm in that frame of mind... back then whenever I felt down I'd make sure I'll be where the nearest party was on Saturday night (laughs) and leave by Sunday, I'm well away..."

Finally, within this theme the matter of 'person's choice' or 'freewill' also seems to colour participants' self-sufficiency. Below, Margaret depicts an experience where she was prescribed medication however she describes her decision to not take them.

"...I must have said something to the GP, because I remember, at one point, he gave me some tablets... and I was working as an auxiliary nurse at the time and I wanted to know what they were. They were Valium and I thought, 'I don't want these'... and so I decided I wasn't gonna take them. I used to work under certain wards and when patients get rowdy and start, they will bring these things, give it to them and they will sort of calm down and sleep and I thought, 'No, I don't want these' (laughs)... I wasn't going to indulge in these things... I thought, 'Oh, these things are tranquilizers; Idon't want them..." 
She further elaborates:

"...I remember, I thought, these things, these are for crazy people, and I'm not crazy. I decided I wasn't gonna go down that road, I wasn't gonna take them..."

And:

"Those things, they don't help; drugs don't help. Just keep you like a zombie; and that's what I don't want; and that's what I didn't want for myself, that's why I wasn 't gonna take anything, you know..."

Although the context to why Margaret spoke to her GP is unclear, Margaret's accounts illustrates how her understanding of the effects of medication and its observed impact on others, strongly influenced her decision to cope with her self-defined depression on her own, and seemingly evade further follow-up with her GP on the matter. Nonetheless, Margaret executed her 'person's choice' or 'freewill'; this choice may have been driven by fear "I'm not crazy" or "just keep you like a zombie", but it was still her decision to make on how to cope with her self-defined depression. To conclude, several aspects expressed by participants within this theme suggests a strong influence that a self-sufficient outlook had on participants' help seeking views in relation to depression, as well as on preferred coping strategies for depression at the time. .

\subsubsection{Participants 'Significant Relationships Experiences in relation to Depression}

This subordinate theme encapsulates the importance of participants' relational experiences with significant others when coping with their self-defined depression and how this impacted on their help seeking views. Participants' accounts highlight significant relationship experiences as key to whether they sought help for the ir selfdefined depression; that is, participants' expressed how important a role, family and close friends play in maintaining a healthy wellbeing when coping with their selfdefined depression, as well as how these relationships impacted on the ir help seeking views. Interestingly, the importance of significant relations hips on help seeking views is also mentioned in the previous two subordinate themes. Furthermore, the influences of cultural norms and family values on participants help seeking views are also echoed in this theme. 
In the excerpt below Margaret describes a need for her depressive thoughts or feelings to be noticed or heard by her family; however this did not occur. When asked what help was available to her at the time? Margaret replies:

“...None because ....I never said... I don't even know if they were and I don't even think that did cross my parents' and siblings' minds that I had those thoughts ...no one stopped to ask me, well, how do you feel about being this way, you know.

Margaret further elaborates:

"I don't know if they ever think that way; being, you know, the society... I don't think it will cross their minds. The way we were brought up, I don't think they thought of your feelings. I don't think anybody ever thought of how you, the individual is feeling. All they could see is that, you've let them down, you know. They never stop to think that; you're thinking, 'Oh, my God! What am I gonna do now?..."

In this account Margaret gives a sense that an individual's feelings were not valued in comparison to the importance of 'family reputation'. What is more, Margaret hints that this reflects an overall cultural perspective "you know, the society, I don't think it will cross their minds". Given her account, it appears if Margaret was given this space she may have been willing to share her thoughts and feelings; Margaret also hints at how doing this might have conflicted with familial or cultural requirements at the time. It is interesting how Margaret reiterates the word "think" in her accounts, which appears to suggest the importance of conscious thought, its impact and having the space allowed to explore it.

Margaret depicts further on other experiences of significant relationships while coping with her self-defined depression:

"Well, no one ever sat down and talked to me; well, I think I was too embarrassed to tell people how I'm feeling anyway. So I never said to anybody. Once I went to my elder sister and I was telling her about certain things that I wasn't happy with and all she said to me, 'well, you don't have to upset yourself'... and I thought, 'Well, you're no help so what's the point of talking to you'. So, I used to bottle things up a lot. I think maybe I was ashamed to talk about certain things..."

And:

“...Then this uncle, I think it was a great uncle, tell him things and before you know it; it's all over the place. I remember, I said something to him once, then I met another relative on the street and she said, 'oh, but I heard so, so and so'; and I was so shocked, I thought, 'No'... and that was when I decided, you know something, keep it. But I never thought of going for professional help as I said..."

In Margaret's excerpts it seems a key influence on her help seeking views was not being noticed or heard within her significant relationships: "Well, no one ever sat down and 
talked to me". In addition, it appeared important for Margaret to experience trust and not feel judged in her relationships: "I was ashamed", "I was too embarrassed" and "before you know it, it's all over the place"; these feelings seemed to strongly influence her views on seeking help for depression. Moreover, as Margaret describes an attempt to open-up to a significant relation, it appeared she felt shut down: "Well, you're no help so what's the point of talking to you"; this appears to suggest the importance of empathetic listening within relationships to aid help seeking positively.

Beverley's account below depicts her experience of being noticed by a family member and how important this seems to be to her at the time.

“... my husband's niece came to visit and realised that I wasn't too well and she used to come and help....she started helping me tidy up...She was young but she was clever enough to say to me to come and show her where to put these things but she did this to help me..... I'll never forget her, yeah... My sisters them were there but I think it was too close. They were too close to me to see what was going on. Not until I told them what the doctor tell me ..."

Beverley further illustrates this with another family member:

“....... I was so pleased that he spoke to my doctor, my husband, in the presence of me ... and by then, my husband start taking notice ... and then he cut down work... he cut down the time at work to do more in the house and he helped, yeah. And eventually, I got out of it because they will help, yes, yes...."

Beverley's excerpts highlights the value of being noticed or heard by significant relations; however, she also emphasises that at times some significant relations may be "too close" to notice. Nonetheless, whether positive or negative both Beverley and Margaret expressed how much a strong influence significant relationships had on their help seeking views when coping with their self-defined depression .

\subsubsection{Participants' Experiences of Health Professionals \& Listening Skills}

This subordinate theme reflects the influences being listened to by health professionals had on participants' self-defined experience of depression and what impact this experience had on the ir help seeking views. In the excerpt below, Beverley shares how helpful being listened to by her GP was on her self-defined experience of depression. 
"....the best thing is for somebody to listen to you when you tell them how you're feeling, listen to how you're feeling and open up to them. But Sometimes... at first I didn't open up. I think, the trigger of the doctor saying to my husband about the medication that I'm taking helped me to tell my husband how I'm feeling more and he kind of noticed, yeah..."

Beverley further explains:

“... my doctor is saying the things that I'm feeling...you see, he's telling him, she's probably gone through this...she's probably gone through this...And we are saying the same thing in different ways and he understood...he was speaking for me anyway.. so, you know... it was good that somebody took over the voice ... you know, so he was my voice. He was good, yeah, he was good, yeah."

Beverley's accounts highlights the importance of being listened to by a health professional and the normalising effect this had on her coping with her self-defined experience of depression. This experience enabled Beverley to open up further to a significant relationship, which seems to echo aspects mentioned within the subordinate theme 'participants significant relationships experiences in relation to depression'. Below, Shirley also shares a similar normalising aspect of being listened to by a health professional:

".... I recognised it because the mid wife mentioned it... because Ijust burst into tears... I was getting ready to get out of hospital and she said oh dear are we having a little bout of depression...so I said... what! do I look like any depressed person to you (laughs) ....she says to me well its common ... I said is it!...so she oh yeah it's quite common after a baby you have a little bout...she says while rubbing my back... so I said I don't really entertain depression so you know it's not common for me!...

Shirley further elaborates:

"...so she said anyway and she sat on the bed and we talk...and it was after talking with her she said never mind but you know you can join a group ... you know find a group...look inside the community hall where you live ...there's lots of groups where mothers meet...you know post-natal depression... so I said I'm not depressed ...I don't have no depression I don't have no depression (laughs) so at least that time I was aware of what was available..."

In this account, Shirley reflects on her self-defined depression experience following the birth of her second child. It seems having a health professional notice, then normalise her feelings "it's quite common after a baby", enabled Shirley to "talk" further about her experience; which in turn gave way to her hearing options about help available "there's lots of groups where mothers meet". This experience emphas is es the important aspects of being listened to by health professionals; furthermore, despite Shirley being reluctant to accept the term depress ion "I don't have depression", Shirley acknow ledges 
her new awareness of existing help "so at least that time I was aware of what was available". Interestingly, Shirley's attitude seems to echo aspects within the subordinate theme ' just get on with it/person's choice'; Nevertheless, this subordinate theme illustrates the strong influences being listened to by health professionals seemed to have on participants' help seeking views and self-defined experience of depression .

\subsection{Overview of master theme two: "....I was depressed...I knew I was depressed"}

The second master theme seeks to explore how participants made sense of coping with their self-defined experience of depression now aged 65 years and over. In addition, the theme examines the influences of these experiences on participants' help seeking views. Three participants reported experiencing the ir self-defined depression aged 65 years and over; however, the context in which each participant's depression occurred varied. Participants also highlighted some issues that had influence on decision-making on whether to seek help or not from mental health services. In this theme participants' experiences with depression are summarised in two subordinate themes: Grief in later life and Depression, and A matter of "inconvenience".

\subsubsection{Grief in Later Life and Depression}

This subordinate theme reflects the influences grief had on two participants' selfdefined experience of depression. Participants now older and reflecting on the ir earlier life experiences of depression described their intentional actions to cope with the ir selfdefined depression. Participants did not discuss any barriers to help seeking at the time.

In the excerpt below, Mary's account describes her self-defined experience of depression aged over 65 years, following the death of her husband four years previously.

"Well yes... after the death of my husband ...it did not impact in me immediately until a year later... one year to the day ... and I woke up.. I was away from home, I was on a retreat and I woke up and the head stone was laid ... and a WhatsApp of it was sent to me and ...I just lost it ... I left the hall of the retreat and went to my room on site, and I remember... just curled up in the foetal position on the bed and I just cried and cried and cried... I had friends that came to my room and I could not be consoled ... prayers were sent up or offered... and.... yeah ...that was my grieving time and it was a dark corner ... really a dark place..." 
Mary further elaborates:

"I knew I was depressed, I knew I was depressed.... and friends that came to the room said that they were very concerned about me because I got a friend whose husband has bi polar ...and she came and said she was really concerned about me ... yeah."

And:

"My faith... yeah I thought to myself... I have a little understanding of what God went through for his son, to have lost his only son ... and I thought Jesus came through it and I will... yeah... and I did."

Mary vividly describes her time of grief. As Mary reflects, she confidently declares twice "I knew I was depressed" and her awareness seemed to be reinforced by the observations and experience of friends. When asked what helped her to cope at the time, Mary reflected on how her Christian faith helped her through.

Like Mary, Beverley connects her self-defined depression experience following the death of her mother three years previously. However, Beverley's account highlights her integral view of being listened to, particularly outside significant relations hips, when coping with her self-defined depression experience at the time.

"Well, maybe grieving is part of depression .... You're grieving; you know why you're feeling like that.... you've lost someone; she's no more there ...and your family them, remember all of you are grieving, you know, every one of you is grieving in different ways... but when you feel that you need somewhere else, you need somebody else to listen to you, Samaritans is the best... for me Ifelt that well, they listen to me and they're not telling anything different from what you know but they help you to understand what you're going through; they help you to make sense of what you're going through and that help...yes...,so outside help is very important."

Beverley further explains:

'Sometimes, your family, they can't see what you're seeing...remember, they're there, going through the same thing in different ways, so it's nice to get somebody's point of view outside of the family; yeah, yeah..."

It is noticeable that Beverley presents part of her account in the third person as if somewhat emotionally distant to her experience. Nonetheless she clearly articulates what she found helpful when coping with her self-defined depression at the time.

Although Mary's and Beverley's experience with grief and depression differed, it seems given their earlier life experiences with their self-defined depression, both Mary and Beverley were able to intentionally seek help be-it 'self-sufficiently' or to source external help. What is more, it also appears that both Mary and Beverley gained the 
knowledge and/or skills to cope with the ir self-defined depression due to the ir earlier life experiences with depression. As a result, this seemed to helped to remove possible barriers to help seeking now aged 65 years and over.

\subsubsection{A matter of "Inconvenience"}

This subordinate theme demonstrates the influence not wanting to "inconvenience" a health professional had on a participant's help seeking attitude when experiencing the ir self-defined depression. Vincent's' account below further illustrates this:

"7 years ago, I was really really really down and I went to see my GP... me and my doctor always get along well.... and we sit down and we talking and talking... and what I really went to see him about, I end up not discussing it.... but I didn't want to walk away and leave the surgery and he may think, say, 'that's madness'.

Vincent further elaborates

"So what you do? You go to get your finger nail clipped; instead of you continuing with what you were going to say....your mind shifts away from that because you don 't want no body to feel you're inconveniencing them..."

Above, Vincent describes feeling really low in mood "down". He appears self-aware enough to seek help from his GP yet when in his GP's presence, seems to find it hard to discuss his low mood "we talking and talking....and what I really went to see him about, I end up not discussing it" due to a concern of "inconveniencing them".

Vincent continues:

"I wanted consciously to express my feelings but nothing made me do it. I don't know why it didn't come out because I have so much confidence in my GP right, that I could tell him anything and he was a dark man like myself so everything was perfect there but that didn't come out.... I think in the long run now, why that didn't come out...I think if I did go down the road of expressing it to my GP, probably he might have put me on some kind of tablets but it might only numb the feelings..... we are always going to our doctors to get something ...but those things can't take away, they only suppress it for a little while, after that, it come back ...."

Vincent's account illustrates his awareness of the change in his mood and his willingness to seek help; yet he was reluctant to follow through in receiving the relevant help. Initially it appeared Vincent was concerned about inconveniencing his GP but when further explored, he revealed his concern with prescribed medication. The issue of stigma around suffering from depression may also have played a role. In addition, Vincent describes an open and positive relationship with his GP, yet the strength of their relationship did not seem enough for him to share his concerns. It may also be possible 
that Vincent held some views on masculinity and on his cultural identity that influence his decision to not tell his GP about his self-defined depression.

Vincent's account also echoes, the matter of 'person's choice' or 'freewill' reported in master theme one. Like Margaret earlier, Vincent too used his 'person's choice' or 'freewill'; this choice may have been driven by the embedded values he held“... those things can't take away, they only suppress it for a little while, after that, it come back....", nonetheless it was his decision to make.

To conclude master theme two, it appears within these themes being self-aware of one's emotions has been key to participants intentionally seeking help; however, as in Vincent case, being self-aware of the change in emotions was not enough to not inhibit help seeking.

\subsection{Overview of master theme three: "You have to decide" Attitudes to Help seeking and Mental Health Service Use}

The third and final master theme captures the primary decisions surrounding participants' help seeking attitudes in relation to dealing with their depression. All participants reported not currently experiencing depression; as a result, some depictions are voiced in the second and third person; interestingly, this may have also acted as a distancing device given the content of the interviews and the associated stigma often connected with mental health concerns. Furthermore, the content of the interviews shows that this may have a particular resonance within the Caribbean community. Nonetheless, all participants highlighted steps to sourcing help for depression. The decisions surrounding participants' help seeking attitudes are coloured by the ir past personal experiences with their self-defined depression as well as their observations and knowledge of their local community.

Participants' help seeking attitudes are summarised into four subordinate themes within the superordinate theme detailed above:

- "Recognise and Start",

- "Why should I go tell somebody my business": Challenges and cultural beliefs,

- "No guarantee": Professional services and 
- "Just get on with it" part 2.

\subsection{1 "...Recognise and Start ..."}

This subordinate theme focuses on the early stages of decision making whilst considering help for depression. Following participant's experiences of coping with their depression, participants described help seeking for depression as a staged process, highlighting a "first" step to decision making. In the excerpt below, Mary depicts what she considers to be the "first" fundamental step to accessing help for depression:

"First of all they got to acknowledge that there is a problem..."

And:

"...You must really want the help... you want to get away from all the negative things that depression brings... you want to step out of that and start anew as if you had a positive side at the beginning and this negative side has taken over then you want to get back to your old self ..."

Mary speaks about the importance of being self-aware ("acknowledge") and desiring help ("want help") as the prime components to seeking or accessing help for depression. In her account she repeatedly uses the word "want" as if to emphasise the desire or need for an individual to "really want help", to "want to get back to your old yourself".

Like Mary, Freddie echo's a similar belief

"The first decision is Ineed change...change of mindset, change of location, change of environment ... you have to recognise and start to apply changing your life ..."

Although worded differently, both Mary and Freddie described an innate awareness and desire for an individual to "change". Freddie articulates how "change" can be needed in various areas of a person's life, yet most importantly there must be initial recognition and desire before accessing help for depression. Interestingly, in the excerpt below, Shirley comments on people not recognising a need for help.

“...some people don't recognise that they need help ... I think a lot of people don't realise that they need help ...sometimes some of them think oh pull yourself together... especially if you're a person who talks to yourself ... and oh I'm feeling a bit down today and maybe don 't know what it is ... they don't know maybe I've been down for the last 2 months and probably need to go and get help..

Above, Shirley acknowledges the need for recognition however expresses she feels many people "don't realise they need help". Shirley seems to echo similar concepts from the "just get on with it" mindset mentioned in Master theme one, stating "some of 
them think oh pull yourself together". It appears from her account, that not recognising the need for help for depression may inhibit help seeking or mental health service use.

Finally, Shirley describes another desire as a starting point:

".The decision they would have to make, is to find out what is causing the depression...that's where they need to start .... but of course they might not be able to start with what's causing it ..... in that case...they need to seek what could help them to be lifted out of their depression ....until they can decide and find out what caused it... find some way some means whether group, support group ... whether it's your pastor, your sister, your parents, your neighbour, your friend .... wherever you know you have somebody....there must be somebody that you have... ...find somebody and sit and talk..

Shirley's depiction above, shares similar ideas to that of Mary and Freddie; that is, there is an underlying desire to seek help, be it to "want help", to "need change" or to find out "the cause" of depression. This desire seems to be the starting point when considering the decisions surrounding help seeking for depression.

What is more, Shirley also draws upon the influences of significant relationships at this early stage. Interestingly, the importance of the close relationships has also been highlighted in Master theme one, as having a key influence on help seeking views. In this subordinate theme some accounts appear distant or prescriptive, as if it is easier to discuss the decisions surrounding help seeking for depression as advice to others rather than to themselves; this may be influenced by cultural views around coping with depression as well as the associated stigma often connected with mental health concerns.

\subsection{2 "Why should I go tell somebody my business?": Challenges and Cultural Beliefs}

This subordinate looks at the dilemmas and challenges formed by participants' internal beliefs and by the beliefs observed within the Black Caribbean community, when considering seeking help for depression. Participants discussed several barriers to help seeking for their depression depicted below. In Leroy's excerpt, he describes how holding on to feelings of shame and embarrassment inhibits help seeking, and how he felt this was particularly prevalent among men of Black Caribbean decent.

“.... You have to decide and set aside some feelings of shame or embarrassment first of all to go and seek help ..... yes for men especially and men of Caribbean descent 
they tend to think that's it's not manly to ... and whatever you got ... whatever feeling ...you can work it out ...you can't tell people your business and so forth especially when there's no blood coming off you ... no cut injury anywhere... you think to yourself... well why should I go tell somebody my business, when I can go and work on it whatever.. that's how we approach it I say ...".

Leroy comments on the influence of possible embedded ideologies or cultural beliefs on a decision to seek help for depression. "It's not manly" and "you can't tell people your business" seem to be internal beliefs that inhibit help seeking. Interestingly, Leroy also compares depression to a cut injury; this comparison appears conflicting to internal beliefs about what is worth seeking help. In addition, Leroy also draws from Master theme one "just get on with it" stating "well why should I go tell somebody my business, when I can go and work on it. Nonetheless, Leroy concludes with this point:

"...Well if you overcome them feelings then naturally you have to think for the betterment of yourself and those around you... It's best to go and do whatever it is you have to go and do to help to recover from it."

Above, Leroy speaks on the importance of overcoming difficult feelings. To "overcome" highlights the strong influences feelings of shame, embarrassment and embedded beliefs have on a decision to seek help for depression. Below, Shirley echo's similarly:

“... it could be a very dark place for some people... some people don't want to admit that they're in that dark place ... a lot of people just keep quiet and that's how they get worse ... ....it's amazing... anything to do with mental health still seems to have that stigma attached where people don't want to talk about it ...so even though you might recognise it and point out to them, they either ignore what you're saying or completely deny it ..."

Like Leroy, Shirley's account illustrates the challenges associated with coping with depression as well as the influences stigma has on inhibiting help seeking for depression Furthermore, although participants acknow ledge recognition as part of the first steps to help seeking (see "recognise and start" theme above), Shirley emphasises that even if self-awareness is evident, this may not be enough to not inhibit help seeking for depression. This emphasis as exemplified by Shirley is also discussed and evidenced in master theme two " a matter of " inconvenience".

Interestingly, Shirley also remarks on her observed cultural differences relating to accessing help for depression:

..... I find the Europeans they handle mental illness differently to us... they are very easy to tell you they depressed... they feeling depressed or what have you... I cannot remember actually hearing any or many from our culture that will actually be 
confessing they are depressed.... Cha I ain't feeling too good ...cha I ain't feeling on top you know today... it will always be in line like that ....I'm feel a little low today or what have you... but to actually come out and tell somebody that you depressed... it's only if that person points it out well you think you depressed or what sort of depressive behaviour is this?.... so I suppose those are things that can stop people especially our people you know from actually seeking help because they don't want to be classified as somebody with depression.

In Shirley's excerpt above, it appears there may be cultural differences within the language used to communicate when one is feeling depressed. "Cha I ain't feeling too good", "cha I ain't feeling on top you know today" or "I feel a little low today" does not appear to be a strong enough indicator for someone to possibly consider seeking help for depression within the Black Caribbean community. What is more, Shirley comments on the direct stigma associated with the term depression within the Black Caribbean community, indicating barriers to help seeking. It is also interesting how Shirley describes personal acknowledgment of depression as "confessing", perhaps indirectly this too highlights the stigma associated with depression, as if it may be shameful to acknowledge to self or others.

Shirley's also considers the cultural aspects of religious belief within the Black Caribbean community, and how this inhibits help seeking for depression.

“... cultural as well... where some people feel that to say your depressed it brings up some demonic interest.... some demon there...... so that's why I think a lot of people don't even share their depression.... and I'm putting my hand up as well I was in that league many years ago.."

Shirley's further elaborates:

“.... With it being .... oh that's a spirit come let us bind and get rid of that spirit (laughs) ....you know no Christian has any right with being depressed so you make such a big thing about it ....cause I remember having friends who was depressed but they wouldn't tell anybody that they were depressed right... ...I can think of one particular person.... when we were talking and as this other person approached she said ...shush ...lets change the conversation because as for this one I don't want this one to start deliverance (laughs).... I don't want this one to start her deliverance on me today..... so we change the conversation (laughs) she said you can't tell people like them your sick much more depressed....".

And:

....as Christians the underlying foundation for them not going for help especially as a Christian is where they feel as Christians they should not be suffering from depression.... and that means something is wrong...either they are not a strong Christian or they're not really a Christian or there is some demonic influence some where there..." 
In the excerpts above Shirley speaks of her own personal experience as a Black Caribbean Christian. She openly shares how thoughts of demonic influences on depression inhibit help seeking. Interestingly, Shirley laughs as she shares her story, perhaps to cover any anxiety from expressing something deeply personal, where she may be left wondering if she would be judged.

Shirley also speaks about her encounter with a friend, who expresses her discontentment with aspects of this cultural religious belief; this results to her friend choos ing to remain silent, therefore inhibiting help seeking. Furthermore, Shirley highlights the possible internal conflicts between religious belief and mental illness, therefore inhibiting help seeking for depression ("either they are not a strong Christian or they're not really a Christian or there is some demonic influence some where there...”).

To conclude, issues highlighted within this theme indicate the strong influences embedded ideologies or cultural beliefs can have on help seeking attitudes. Furthermore, issues highlighted also indicate inhibiting beliefs to help seeking for depression.

\subsection{4 "No guarantee": Professional Services}

This subordinate theme explores the challenges participants expressed, when considering looking for help from professional health services for depression. In the excerpt below, Leroy explains his reluctance to seek help as he becomes suspicious of health professionals' intentions.

“.... well when I'm thinking about being referred I become slightly suspicious.... that is, that the person who is doing it is doing it for the wrong reason, maybe to get a target done or whatever....rather than seeing that your well-being is looked after....."

Leroy further elaborates:

“...Well for example... some years ago I had a small inclination of hernia... and I just manage to mention it, I wasn't in no pain, I wasn 't in no hurt a little elevation of the skin area and before I know it I was under the knife somewhere.... and now I have a bigger problem with it...now I don't feel like going back because of the suspicion that I have....It's not that they don't care but there is a target somewhere to get certain things done , certain numbers up ... well I heard somewhere..."

It seems Leroy's expectations were not met following his visit to a professional health service. This appears to have created distrust, to the extent where the experience may have left him conflicted or rather reluctant to seek further help from professional health 
services for depression ("I don't feel like going back because of the suspicion"). Below, Shirley depicts a similar experience where she describes a lack of trust to inhibit accessing help from professional health services for depression.

“...I supposed a GP could be a good port of call ...it is just I don't trust them where I am at my surgery there because if they can't help you with medical issues I won't trust them with my mind (laughs)..."

Shirley jovially expresses her lack of trust with seeking help from her GP. Like Leroy, this viewpoint, stems from the dissatisfaction following her own personal experiences. Although both Leroy and Shirley experiences where not mental health related, it demonstrates just how lasting and influential an experience with a health professional is when considering future help seeking. In addition, their depictions evident that poor experience within professional health services may inhibit future helping seeking for health services, even it is an unrelated health matter.

Conversely, in the excerpt below, Earl expresses the influence appointment waiting times has on the decision to access help from professional health services for depression.

“...sometimes they just give you an appointment for about two weeks before you could see them. ...sometimes three weeks, you know what I mean because no guarantee ...if you want to go and see your GP, you have to go inside there for 8 $o$ 'clock in the morning ....you will be seen that day but you won't be seen the same time when you go there and then they tell you come back 4:30 in the evening or 5 O', clock in the evening, you know...otherwise if you just make an appointment over the phone, you won't be seen until about two to three weeks.

Mary echo's a similar concern

"... you know with all the cut backs, now my concern is the long waiting .. If they are seeking quick help you know... all the bureaucracy and the long waiting ..."

Above, participants express concerns of long waiting times for appointments as deterrents from seeking help from professional health services for depression. A pattern surrounding the lack in confidence with services seems to weave through this theme, and in turn, indicate strong influences to inhibiting help seeking for depression from professional health services.

Finally, within this theme the matter of 'misrepresentation' within professional health services is highlighted as another concern and a considerable influence on help seeking for depression. Below, Vincent elaborates further on this. 
"...I think the way the care system set up in England and Wales; within that, you should be able to find an advocate who can represent you because a lot of what's going on, we are misrepresented. I have very strong views of that; a lot of things are going on in our lives as black people in this country, we are misrepresented ..."

In this excerpt Vincent shares his views on the importance of advocacy where required when seeking help for depression. He emphas ises on being "represented" through this process, and openly shares his views that "Black people" in this country are "misrepresented". He suggests misrepresentation as a possible deterrent to accessing help and alludes to racism or discrimination, although this is not actually named.

\subsection{5 "Just get on with it" Part 2}

This final subordinate theme demonstrates the influence a self-sufficient outlook has on decisions surrounding accessing help for depression. As in Master theme one, participant's accounts following their experiences with depression gave a sense to "just get on with it" and to rely on their own resources as a preferred coping strategy for depression. Margaret's excerpt below further illustrates this:

“... Well, as I said, I didn't go about seeking help apart from I spoke to my older sister and what she said to me, I didn't find it helpful so it was more of self-help for me.... '. I decided I'm going to get on with things, stop worrying about these things... they are always there at the back of my head, even now, you know... but if anybody, if any of my sons, daughter-in-laws, grand children or anybody come to me and say something, I'll try to help them and I know I'll now say, ....you need to go and see a counsellor? how far down are you?...but for me, I never thought of going for help anywhere..."

Compared to her earlier years, Margaret now older seems more aware of the services to direct someone needing help with depression to. It also appears given Margaret's past experiences with coping with depression, Margaret appears to be be ing more inclined to "get on with things" rather than seek help for herself as her preferred coping strategy for depression. This seems similar to the subordinate theme discuss above where past experiences within professional health services influence future help seeking for depression.

In the excerpt below, Leroy highlights the influence of his parent's self-suffic ient advice strategies on his decisions to seek help for depression.

"...What would stop me from seeking help is if Ifeel in control of it and it is something that I can deal with then I would not go forward for any help ...because according to our parents back then... there were lots of things that they showed us that could be done 
to avoid certain situations .. sickness wise and so on so ..... So if I can do that before any approach to anything then I will..."

Shirley echo's similarly about parenting

“....another thing.... people who have been brought up with this stiff upper lip and... pulling yourself together.... and it's weak... you got to be strong... that's another thing that stop people.... their upbringing...the way they were brought up...what they heard and what they equate to weak and strong... so admitting that you are depressed could be known as weakness in you as oppose to.. you know something I'm feeling a little low, let me go and get some medication or let me go and at least talk to somebody about it ..."

Both Shirley and Leroy discuss the effects of engrained beliefs from childhood "upbringing" on help seeking attitudes; indicating the considerable influence learnt behaviour or attitudes has on help seeking for depression. It seems if learnt behaviour or attitudes from parenting accentuated coping with depression independently, individuals are less likely to seek help externally. In addition, interestingly depression be ing seen a "weak" vs "strong" draws similarly from depictions noted in the subordinate theme challenges and cultural belief.

\subsection{Summary of Results Chapter}

This chapter provided a narrative of eight Black Caribbean older adults' experiences and understanding of coping with depression and the influence the ir experiences had on their help seeking views. Participant's narratives illustrated the varied influences personal experiences and cultural beliefs have on help seeking views and preferred coping strategies for depression. This first master theme 'If you don't know, you don't seek help" sets the context for the proceeding themes. We learn how in earlier life participants made sense of coping with their self-defined depression and what influence this experience had on their help seeking views.

The second master theme "I was depressed...I knew I was depressed" explored how participants now aged 65 years and over made sense of coping with their self-defined experience of depression. Several issues were discovered to impact on participants' decision to seek help or not with depression.

The third and final Master theme "you have to decide": Attitudes to help seeking and mental health service use captured the primary decisions surrounding participants' help seeking attitudes, when considering seeking help with depression. Although participants reported not currently experiencing the ir self-defined depression, we 
discover a varied process, one influenced by several facets to the decisions surrounding help seeking for depression. 


\section{CHAPTER FIVE: DISCUSSION}

\subsection{Chapter Five Overview}

Chapter five will discuss the main findings derived from the analysis, which relates to the current study's research questions and existing literature explored in chapter two. Implications for Counselling Psychology and clinical practice will be outlined, and the strengths and limitations of this study examined. To conclude, the chapter will close with recommendations for future research.

\subsection{Reflection of the Study's Aims}

The aims developed for this study are:

- to explore the personal experiences, meanings, and contexts that underlie help seeking in relation to self-defined depression among the UK's older Black Caribbean adults, both now and when younger.

- to explore some of the barriers experienced by participants, if any when considering seeking help from mental health services.

In line with these objectives, the research questions developed are:

1. How do older Black Caribbean adults make sense of coping with (self-defined) depression?

2. How can we better understand the decisions that older Black Caribbean adults make surrounding help seeking and accessing mental health services in relation to depression?

\subsubsection{Overview of Key Findings}

Three master themes emerged from the analysis:

1) "If you don't know, you don't seek help"

2) “...I was depressed...I knew I was depressed" 
3) "You have to decide": Attitudes to help seeking and mental health service use.

Overall, the findings in this study revealed how participants past personal experiences of coping with depression including migratory histories, cultural and religious views, and personal relationships influenced help seeking views and preferred coping methods for depression. In addition, several issues were discovered to influence dec is ion-making on whether to seek help or not from mental health services for depression. Findings from this study could be considered to inform the delivery of mental health services and be transferred into policy.

\subsection{Research Question One}

\section{How do older Black Caribbean adults make sense of coping with (self- defined) depression?}

\subsection{1 "If you don't know, you don't seek help"}

Research question one sought to explore and contextualise how older Black Caribbean adults in this sample, cope with self-defined depression. Findings from master theme one sets the scene for the study's preceding the themes, revealing how in earlier life (pre-age 65 years) participants made sense of coping with their self-defined depression and what influence their experiences had on their help seeking views.

\subsubsection{Coping with Depression}

In order to better understand the coping strategies for self-defined depression, results from this study suggests the importance of mental health professionals considering influences of previous life experiences of coping with depression from service users. Research has shown that the type of coping an individual employ depends on various subjective components, which includes whether a person decides to use a particular strategy or not (Lazarus and Folkman, 1984). With this in mind, and in line with the principles of this study (to understand experiences, meanings and contexts of individuals (Yardley, 2000)), this study explored the various experiences participants described across their life span as having a strong influence on how they made sense of 
coping with self-defined depression and what impact this had on their help seeking views. .

\subsubsection{Reactive Depression vs Endogenous Depression}

Earlier in life, all participants described living through difficult times, and associated that stage of life with their self-defined experience of depression. Participants' experience of depression seemed to be reactive rather than endogenous; which means, participants' experience of depression followed either a stressful or traumatic event, rather than it being of biological influences. Nevertheless, as discussed earlier, all participants at the time of interviews did not report current experiences of the ir selfdefined depression. The fact that there was no active report of depression at the time of interviews is not surprising; as research has shown many older adults to have good mental health and live meaningful, rich lives (World Health Organisation (WHO), 2016). In this study, all participants, having lived through and experienced difficult times, had successfully navigated through life; and in turn, demonstrated immense resilience (Centre for Policy on Ageing, 2014).

\subsubsection{Participants' Experiences of Migration in relation to Depression}

Common among participants' previous life experiences of coping with depression, was their profound recognition of the relationship between them migrating to the UK and their first experience of self-defined depression. During this time, participants discussed not know ing where to access help and not having the language/ terms to name it. This lack of know ledge may be seen as a barrier to help seeking, and could possibly be termed as 'mental literacy' (The Sainsbury Centre for Mental Health (SCMH), 2002). However, this term generally speaks of the absence of information surrounding mental illness rather than of services in mental health $(\mathrm{SCMH}, 2002)$. In addition, the lack of knowledge described by participants also assumes that there is one dominant and overarching model of depression and intervention.

Over decades, research has documented the impact of previous mental health service experience or knowledge of mental health services on mental health access for older adults. Studies have argued that the low uptake of mental health services from this group may be due to people not ever having to access services previously. In addition, older adults with migratory histories may not know the role of the GP as a source of information and support for mental or emotional problems (Jacob et al., 1998), or they 
may find it difficult to understand the services available and how to access them (Bhui and Bhugra, 2002). Interestingly, the current study echoes the same, sugge sting the need for wide-spread promotion of 'how' 'why' and 'where' to access services among the older adult population.

The research findings also illustrate specific attitudes at the time of participants migrating that may be seen as a barrier to help seeking. The "opportunity" to come to the UK to better one's self with "work" seemed to over-ride desires to seek help specifically for their self-defined depression. Many participants described prioritising adapting to a new way of life in the UK, resulting in "not looking for help" outside of this focus - regardless of the after effects (experience of self-defined depression) of migrating. Consequently, if participants have learnt earlier in life to dismiss feelings relating to their self-defined depression, it can be argued that now that they are older, the urge and/or behaviour to seek help may not exist.

\subsubsection{The Influence of Culture on Coping in relation to Depression}

Participant's help seeking views seemed to be heavily influenced by their 'cultural norms' at the time of the ir self-defined depression experiences. Research has shown the construction of help seeking attitudes to be underpinned by a person's culture (Bhugra and Gupta 2011; Bhui 2013). Likewise, the methods people use to preserve a person's psychological stability are partly established and defined by cultural, societal and health rules; as well as the meanings which individuals assign to them (Fernando, 2014; Tribe, 2014). In this study, findings highlight a community mind-set/attitudes and the impact of this on help seeking views and coping for depression.

Research has provided evidence that Black Caribbean adults ge nerally do not perceive consultations at the GP as a way to get help for depression; instead they 'getting on with things' as a method for managing emotional pain(Lawrence et al., 2006). In this study, many participants' gave a sense of "just get on with it", as if there was no other choice but to deal with ones experiences on their own. To "just get on with it" also seemed to be motivated by a sense of being "depended on" by participants families; suggesting also the strong influences of cultural or family values on help seeking views . Furthermore, the need to provide or care for one's family independently seemed to be key to coping well with participants' self-defined experience of depression. 


\subsubsection{The Influence of a Self-Sufficient Outlook on Coping in relation to Depression}

Self-sufficient approaches to coping with self-defined depression appeared common among participants; that is, participants preferred to source help from within themselves instead of seeking out professional help. Participants reported strategies such as "escapism" through music, or simply sourcing another activity to do. Findings in this study suggests that although older B lack Caribbean adults may not be seeking help from mental health professionals, they still hold proactive strategies of coping that may be used when experiencing their self-defined depression. Previous studies similarly suggests the same, where it has been found that people regularly sourced alternative help (i.e. not from psychologists) for their mental health concerns from networks (i.e. family or friends), who did not offer scientific interventions' (Rickwood et al.,1994; Rockwood et al., 2007). In addition, 'freewill' or 'person's choice' also seemed to colour participants' self-sufficient approaches, and in some cases acted as a barrier for help seeking. For example, participants' perceptions about the negative and damaging side effects of treatment influenced their decision-making on whether to seek help or not from mental health professionals, including their GP for self-defined depression.

\subsubsection{Participants' Significant Relationships Experiences in relation to Depression}

The study's findings highlighted relational experiences with significant others as key to participants coping with their self-defined depression. Research has shown that relationships and social support has been identified as one in many aspects affecting the wellbeing of older adults (Age concern and The Mental Health Foundation 2006).Other aspects identified to affect the well-being of older adults are: physical-wellness; being poverty-stricken; being discriminated against; relationships and social support; and involvement in meaningful activities (Age concern and the Mental Health Foundation 2006).Participants reported how important it was in significant relationships to not feel judged when talking about their self-defined depression. Participants' also highlighted the positive impact of being listened to in these relationships and how this enabled help seeking with the ir self-defined depression. Findings in this study also emphasised the importance of empathetic listening within relationships and how this positively enables help seeking for self-defined depression. 
On the whole, a number of studies have noted the importance of significant relationship experiences (including social networks) as a mediating aspect on improving mental health (in particular with depression) and wellbeing among the older adult population (Cornwell and Waite 2009). As a result, mental health professionals may need to consider strategies to help older people make best use of their relationships/social networks to maintain wellbeing (preventative measures) or as a way to gain access to mental health services when required. In addition, mental health professionals may also consider social prescribing as a way to maintain wellbeing, and/or enable better access to mental health services for the older adult population. Importantly, in line with the values of Counselling Psychology (Karademas, 2009), social prescribing strives to address an individual need in a holistic way, as well as empower them to take control of their own health (The Kings Fund, 2017).

\subsubsection{Stigma and Beliefs in relation to Depression}

Some studies on older adults have found their beliefs surrounding mental health problems as shameful, with some older adults believing the ir mental health problems should be concealed from everyone - including health professionals (IAPT, 2009). In this study, participants held thoughts such as: "I was ashamed", "I was too embarrassed". Generally, research has shown that older adults have a heightened awareness of stigma relating to mental hea th and that they are frequently considered by others to have negative attitudes to looking for help for mental health concerns (Mackenzie et al., 2006). For these reasons, mental health professionals may need to work closely with this population to help reframe shameful, stigmatising beliefs held regarding mental health, as evidence has shown this to strongly influence their attitudes to seek help or not for depression.

\subsubsection{Participants' Experiences of Health Professionals and Listening Skills}

Research findings in this study have also reinforced how effective mental health provision rests upon good communication and meaningful interactions between service users and mental health providers (Wills and Holmes-Rovner, 2006). Participants' in this study reported that "being listened to" by health professionals significantly enables help seeking. This discovery also supports previous research conducted by Mulvaney et al., (2011) in the USA, where service users also reported "being listened to" by health professionals as effective in coping and help seeking. Specifically, African American 
service users in their study, emphasised the listening skills of health professional as key to feeling understood and successfully helped (Mulvaney et al., (2011). All in all, although the USA holds a different context in regards to public health services, UK mental health professionals can still ga in some insight on how to reduce discrepancies in health and mental health service delivery for a diverse population.

\section{4 "....I was depressed...I knew I was depressed"}

\subsubsection{Coping with Depression Aged 65 Years and Over}

Three of the eight participants reported experiencing their self-defined depression aged 65 years and over. Given participants earlier life experiences with their self-defined depression, participants were now able to clearly recognise signs of recurrence aged 65 years and over "I knew I was depressed". Participants also highlighted some issues that had influence on decision-making on whether to seek help or not from mental health services.

\subsubsection{Grief in Later Life and Coping in relation to Depression}

Participants in this study associated grieving as a key aspect of their self-defined experience with depression; in particular, this was subsequent to the passing of a significant other. Although research has shown many older adults to have good mental health(WHO, 2016), having lived through and experienced difficult times (Centre for Policy on Ageing, 2014); studies have also found this population to be more susceptible to mental concerns relating to significant life events such as: partner bereavement, retirement, the onset of a long-term health condition etc. (Tribe, 2017). Nonetheless, many older adults have demonstrated immense resilience during these difficult times (Centre for Policy on Ageing, 2014).

\subsubsection{Religion, Spirituality, Faith and Coping in relation to Depression}

Participants' also reported how their spirituality or faith helped them to cope with their self-defined depression. Faith and religious practices such as prayer have been recognised as significant methods of coping among members of the UK's Black Caribbean community (Lawrence et al., 2006). For these reasons, Counselling Psychologists and other mental health professionals may find it helpful to encourage UK older adults to talk about their faith and beliefs even if the mental health professional initially feels uncomfortable with this (Tribe, 2017). In addition, research 
has suggested that religious, spiritual leaders or healers may be thought of as better suited and a sufficient base of help, rather than first-w orld models of therapeutic and/or psychiatric care for some members of BAME communities (Lawrence et al., 2006).

\subsubsection{Shared Experiences and Coping in relation to Depression}

The findings further showed how the friendships participants held with people who also have previous experience of depression, helped them to cope with their self-defined depression. Participants revealed how the overtly expressed concerns of friends, as well as being listened to by them, helped participants to cope with their self- defined depression. In line with previous research, the findings in this study indicate the importance of previous experiences with mental health services may have on coping and mental health service use (Jacob et al., 1998; Bhui and Bhugra, 2002; Bhugra et al., 2004).

\subsubsection{A Matter of "Inconvenience"}

Participants' reported not wanting to "inconvenience" mental health professionals as a barrier to help seeking. Research has shown that Black Caribbean adults generally do not perceive consultations at the GP as a way to get help for depression (Lawrence et al., 2006). In line with previous studies, the current study suggests a similar perception and highlights the need for wide-spread promotion of 'how' 'why' and 'where' to access services. All in all, the findings relating to question one demonstrates the various subjective components of coping with self-defined depression among this sample of older Black Caribbean adults living in the UK. Furthermore, some issues relating to decision-making on whether to seek help or not from mental health services were also revealed.

\subsection{Research Question Two}

How can we better understand the decisions that older Black Caribbean adults make surrounding help seeking and accessing mental health services in relation to depression? 


\subsection{1 "You have to decide": Attitudes to Help Seeking and Mental Health Service Use.}

Research question two sought to capture the primary dec isions surrounding participants' help seeking relating to mental health services for depression. Although all participants reported not currently experiencing their self-defined depression, all participants highlighted steps to sourcing help for depression, and discussed barriers as well as enablers to decision making. Participants' thinking surrounding decision making appeared to be coloured by participants' previous personal experiences with the ir selfdefined depression; as well as their observations within their local community.

\subsubsection{A "First Step" to Help Seeking in relation to Mental Health Services for Depression}

The research findings illustrated a "first step" to decision making, when considering help seeking for depression. Participants described this decision making as a "process". To begin, participants reported the "first step" as having to be self-aware ("acknow ledge") and desiring help ("want help") as the prime components to seeking or accessing help for depression. Participants described an innate awareness and desire to "change". This awareness and desire to "change" can be seen as an enabling contributor for help seeking. Participants also recognised that many people "don't realise they need help", and highlighted this as barrier to help seeking re lating to mental health services for depression.

\subsubsection{Influe nce of Significant Relationships on Help Seeking in relation to Mental Health Services for Depression}

During the early stages of decision-making, participants described significant relationship experiences as key to help seeking relating to mental health services for depression. These findings are consistent with the number of studies that have evidence of how important a role close friends and family play in the wellbeing and health of older adults (Cornwell and Waite 2009). Importantly, these findings are also in line with the principles of Counselling Psychology, which considers the impact of contextual factors such as social support, relatives and friends (Karademas, 2009). For these reasons, when working with this client group, Counselling Psychologists and other mental professionals need to hold in mind the positive impact of the service user's wider-network in recovery. 


\subsubsection{Stigma, Beliefs and Culture on Help Seeking in relation to Mental Health Services for Depression}

As part of the early decision-making process, participants emphasised the need "to decide and set aside some feelings of shame or embarrassment" prior seeking help. This supports IAPT (2009) report which states feelings of shame and embarrassment to have influence on a decision to seek help for depression. In addition, the current study also highlighted how stigmatising the term depression is within the Black Caribbean community: "those things ... can stop our people (Black Caribbean people) you know from actually seeking help because they don't want to be classified as somebody with depression".

As discussed in question one, findings in this section also highlighted the strong influence personal beliefs (or assumptions) and the beliefs (or assumptions) held within a community have on an individual's decision to seek help or not - including mental health service use (Fernando, 2014; Tribe, 2014). In particular, findings for question two revealed the strong influence internal conflicts between religious beliefs and mental illness had on a person's decision to seek help or not. Participants further highlighted the need for an 'intentional action' to set aside restrictive beliefs in order to enable help seeking relating to mental health services for depression. Given these findings, Counselling Psychologists and other mental profess ionals could support older Black Caribbean adults with restrictive beliefs (or assumptions) to reframe thinking and develop a healthier approach.

\subsubsection{A Matter of 'Choice'}

Although participants described 'recognition' as part of the first steps to help seeking, there was a consensus that this may not be enough to not inhibit help seeking. This links with discussions in question one, where coping has been identified as subjective, which includes whether a person decides to use a particular strategy or not (Lazarus and Folkman, 1984). For these reasons, the decision to seek help or access mental health services for depression depends on the individuals' experiences and meanings they attribute to the feelings and/or behaviours they identify. 


\subsubsection{The influence of Language on Help Seeking in relation to Mental Health Services for Depression}

The research findings revealed how the language or terms used to communicate feeling depressed differed among people and may not overtly indicate that someone needs to seek help or access mental health services for depress ion. "Cha I ain't feeling too good", "cha I ain't feeling on top you know today" or "I'm feel a little low today" appeared to be terms often used to described self-defined depression among older Black Caribbean adults; however, this did not appear to be a strong enough indicator for someone to consider seeking help for depression. As discussed in question one, research has shown the construction of help seeking attitudes to be underpinned by a person's culture (Bhugra and Gupta 2011; Bhui 2013), and the meanings which they assign to them (Fernando, 2014). For these reasons Counselling Psychologists and other mental professionals understanding the various language or terms used to communicate when feeling depressed, could help to improve material used to promote services and strengthen therapeutic alliances.

\subsubsection{Previous Experience with Health Professionals in relation to Depression}

The study's findings also found the perceptions participants held following previous experience with health professionals or services to strongly influence the decision making process to seek help or not for depression. Research has shown previous experience of treatment or mental health service use as a strong predictor of service use among older adults (Conner et al., 2010). In this study participants reported being suspicious of health professionals' intentions, and expressed concerns about the long waiting times for appointments as deterrents from seeking help from professional health services - including mental health.

On the whole, in this study participants dissatisfaction following previous personal experie nces of health professionals' seemed to have created distrust, to the extent where participants were left feeling conflicted as to whether to seek further help from professional health services for depression. The findings indicate poor experience within professional health services to inhibit future helping seeking for health services; even if it is an unrelated health matter. What is more, it demonstrates just how lasting and influential an experience with a health professional is when considering future help seeking. As a result, Counselling Psychologists and other mental health profess ionals 
will need to think together with this population about how to create better systems of service delivery. The findings also indicate how important it is for services to appear and be reliable in order for service users to seek help or access mental services or not.

\subsubsection{The Influe nce of Childhood on Help Seeking in relation to Mental Health Services for Depression}

To conclude question two, the findings demonstrated how beliefs/culture views formed from childhood "upbringing" strongly informed the decisions surrounding help seeking relating to mental health service for depression amongst participants in this study. Findings suggest that individuals are less likely to seek help externally if learnt behaviour or attitudes from childhood accentuated coping with depression independently. As our past colours or influences our here and now; this finding not only sets the context for answering question one, but also concludes the answers for question two. This finding can also be seen as a thread throughout this discussion chapter. In summary, research has shown that coping is subjective (Lazarus and Folkman, 1984) and is multi-faceted. It may also be influenced by a person's culture and beliefs (Fernando, 2014; Tribe, 2014) .For these reasons, when considering how to better understand the decis ions surrounding older Black Caribbean adults help seeking relating to mental health services for depression, it becomes evident how earlier life experiences inform current coping strategies and help seeking views relating to mental health services for depression.

\subsection{Implication for Counselling Psychology and Clinical Practice}

\section{6a Service level}

As the ageing population is rising in number, mental health services for older adults need to be expanded and adequately resourced to ensure that they meet the needs of the entire population including older Black Caribbean people. The findings in this study show how older Black Caribbean adults are currently not utilising mental health services; as a result, policy needs to ensure that services are developed to meet the needs of this group and the entire population through consultation and community engagement. Findings in this study suggests policy makers may need to ensure information about mental health and relevant available services are made widely 
accessible, and that health promotion activities may need to be undertaken. Some ways of achieving this but not limited to are: developing focus groups, hosting coffee mornings, having stalls at community events, building close relationships with faith and other community organisations where this population regularly congregate.

The need for community engagement within mental health services is being increasingly recognised nationally and internationally to improve services and ensure they meet the needs of all members of our communities (NICE, 2016). However, reports have found community consultation and engagement are often not carried out effective ly or accurately (Lane \& Tribe 2010; NICE, 2016). This discrepancy highlights the need for frequent consultation and engagement. Importantly, the need for consultation and engagement aligns with the findings in this study, as findings show how older Black Caribbean adults welcome engagement from health professionals, while highlighting being listened to as a positive contributor to enabling help seeking for depression from mental health services. Given these findings and building upon NICE, (2016) guidelines for community engagement, policy makers can consider utilising the rich source of information which is held by the older Black Caribbean community and use this in planning services.

Consultation and community engagement has specific resonance when groups which have been defined as hard to reach are considered (Tribe \& Bell, 2018). The importance of listening to communities and community organisations is also being increasingly recognised as part of a process that can reduce health inequalities (WHO, 2020), enhance the quality of services (NICE, 2016) and contribute to enhancing accessibility and appropriateness (British Psychological Society, 2018). Mental health providers need to comprehend the decisions and experiences of service users or potential service users to understand and plan appropriate service provision, and to deliver effective, culturally appropriate and accessible services (Lawrence et al. 2006). The current study supports these guiding principles and gives recommendation for policy makers to ensure community consultation and engagement is carried out effectively and appropriately with older Black Caribbean adults to help aid help seeking in relation to mental health services for depression.

The evident concerns surrounding mental health service use from Black and ethnic minority older adults, highlights the need for mental health providers to try their best to guarantee that services are accessible, suitable and pertinent to the populations they serve. As a result, mental health providers need to actively plan services with older 
Black Caribbean adults along with their carers (when appropriate) to identify gaps in present-day service provision; In addition, planning services collaboratively enables mental hea th providers to deliver more effective, culturally appropriate and responsive services (NICE, 2011; Tribe, 2017).

Visiting the GP was recognised as a pathway to accessing mental health services for depression; however, it was not always seen as most appropriate by the older Black Caribbean adults in this study. Some reasons found in this study to inhibit help seeking from the GP are: distrust and not feeling listened to by the GP. As a result, GPs' might benefit from additional training relating to working with this population. In addition, Counselling Psychologists and other mental health professionals could consider other pathways to care which might be an alternative to going to the GP; for example, mental health providers could consider an option for this population to also self-refer as a possible way to enable easier access to services or help seeking (Brown et al., 2010).

Mental health services for older adults should also be integrated with community services in order to make help seeking more accessible and services more appropriate for Black Caribbean older adults. To help facilitate access to psychological support, Counselling Psychologists and other mental health profess ionals could work together with this client population in a non- stigmatising or generic community setting around the people they may trust. In addition, the knowledge gained from working with communities who deliver support to this client group enables mental health services to learn from this population, develop appropriate professional interventions and help build up the community resources already in place. What is more, this may also be an opportunity for mental health providers to identify practical steps to encourage early access (SCMH, 2002), especially as previous research show that Black Caribbean people in UK to delay seeking help, and instead present to mental health services in serious states of crisis ( $\mathrm{SCMH}, 2002)$.

Importantly, joint working among multi-disciplinary services does exist, and studies such as Morganet. al, (2009) on Black and other ethnic minorities in London have already helped to develop methods of working that were compatible with each community. Furthermore, findings from this current study highlighted aspects key to wellbeing (i.e. Spirituality and religion), which Morgan et. al, (2009) also recommended to be integrated when working with this client group. However, it is key to note that while it is important to look at the older adults cultural and spiritual needs, 
the wider health inequalities such as social exclusion must be addressed as these factors could exacerbate any untreated mental problem (Department of Health (DoH) 2005).

A number of studies have recommended the further training of mental health professionals on the cultural appropriateness of services. However, mental health providers need to ensure that training and practice provided are relevant and appropriate to the community they serve (Fernando, 2004). Policy recommendations about Consultation through community engagement as discussed earlier in this section can support this process. Through training, Counselling Psychologists and other mental professionals can improve skills working across different cultures (Patel et al. 2000) and gain a better understanding of the specific influencing cultural norms, which can inform service development. However, in doing so mental health providers need to find an appropriate balance in delivery to ensure that training is not tokenistic but instead be part of a wider and systematic approach to service delivery (Tribe, 2017).

\section{6b Clinical level}

Counselling Psychology tries to take into consideration the impact of contextual aspects (i.e. relatives, friends and social support) on a persons' life (Karademas, 2009). This study has emphasised the value of holding a holistic approach when working with older Black Caribbean adults; as well as the importance of working with this populations' wider network when in recovery. For these reasons, the holistic approach becomes key to better understanding the coping strategies used or help seeking views/attitudes formed by an individual.

Given the above view, Counselling Psychologists and other mental health professionals' may find it helpful to encourage this population to talk about their earlier life experiences relating to he lp seeking or coping with depression. Counselling Psychology promotes empowerment and emphasises building a person's strength (Karademas, 2009); therefore, by older Black Caribbean adults clients talking about their earlier experiences of coping with their self-defined depression, clients may be able to build upon the helpful skills they have acquired across the life span, to help cope with depression. Furthermore, if unhelpful strategies or attitudes are found during treatment, mental health professionals can help clients to reframe thinking or learn healthier methods of coping with depression. 
Faith and religious practices such as prayer have been recognised as important methods of coping among members of the UK's Black Caribbean community (Lawrence et al., 2006). In this study it has also been identified as an essential resource and source of strength; as a result, Counselling Psychologists and other mental health professionals may find it helpful to encourage UK older adults to talk about their faith and beliefs, which in turn may strengthen resilience and strengths already instilled. On the whole, research has shown that in therapeutic work spirituality can be a rich resource (Dein et al., 2019). Therefore, where necessary, Counselling Psychologists and other mental health professionals may need to be trained so that they are equipped in ways to attend to ideas surrounding spirituality and religion.

The current study also found the relationship and communication between service user and mental health professional as key to help seeking and access to mental health service use among older Black Caribbean adults. Therefore in line with the National Institute for Health and Clinical Excellence (NICE), 2011 guiding principles on improving services users experiences in Mental Health services, Counselling Psychologists and other mental hea lth professionals need to listen and take time to build trusting, supportive, empathic and non-judgemental relationships as an essential part of care. In addition, help offered needs to be offered in an atmosphere of hope and optimism.

To conclude this section, consultation and community engagement with older Black Caribbean adults is imperative. The current study has highlighted this as key for this population to improve help seeking and access to mental health services in relation to depression. On the whole, consultation and community engagement can support relevant and appropriate changes to clinical practice and service provision. In doing so mental health professionals may also avoid stereotyping, consider culture appropriately and recognise the uniqueness of human experience (Tribe, 2017).

\subsection{Strengths and Limitations}

The researcher recognises several strengths and limitations to this study. The study acknowledges that key to Counselling Psychology are the personal experiences of an individual (service user) and the meanings attached to them; this is to help Counselling Psychologists to provide appropriate help and support (Rennie, 2007). For this reason, 
the current study applied a qualitative framework. Qualitative analysis is considered a strength of this study as it allows for rich data and for an in-depth exploration of participants experiences of coping with depression. The qualitative framework also helps us to better understand the decisions that older Black Caribbean adults make surrounding help seeking relating to mental health services.

There is a scarcity of qualitative studies that examine the experiences of older Black Caribbean adults and the ir perceptions of barriers faced when looking for help from mental health services. This study has specifically helped to highlight some aspects that influence help seeking for this population; as well as the coping strategies used for depression among the older Black Caribbean population in the UK. In addition, the research questions in this study were addressed and findings from this study were relatively supported by existing literature. Although several studies have illustrated the effect cultural ideas about mental illness have on attitudes and/or behaviours to seeking help in relation to mental health services (Furnham and Chan, 2004); these studies have mostly taken a quantitative approach. Therefore, through the use of qualitative analys is, this study has contributed to enhance the critical depth of these studies. What is more, the research findings revealed areas for further exploration.

The researcher being of Black Caribbean decent is also considered as one strength of this study. The researcher found this aspect helped to build an alliance during the interview ing process. In addition, the researcher found this aspect to also potentially a id participants to be comfortable when being questioned and be open with their narratives. Given the researcher's familiarity with this client group this could also possibly be seen as a disadvantage; however, in line with philosophies of IPA (the ability to "bracket off" the knowledge and experiences of the researcher, in order to increase ideographic understanding of phenomena (Smith et al., 2009), the researcher reflected on personal relevant experiences and used supervision to explore it.

It is possible that during the interviews, participants may not have been completely transparent with the researcher due to the fear of being judged. Therefore, analys is may not reflect participant's views in its entirety on help seeking and coping with depression. Nevertheless, although perspectives shared may not reflect accounts in its entirety, some insights into the UK's older Black Caribbean adults have been shared. What is more, IPA is subjective therefore truth statements may be seen as tentative (Smith et al., 2009). 
Research questions may have possibly been influenced by the researcher's own knowledge, experiences and beliefs. However, if another researcher with dissimilar experiences conducted this study using IPA, the data could reveal a different set of themes. Nevertheless, the process of co-constructing meaning is crucial to this analys is. Furthermore, the researcher remaining aware of their biases as a means of reducing personal and professional impact on the data is also vital to the analysis process. Generally, there are a number of criticisms relating to qualitative research and the methods used. For example, there are criticisms concerning the researcher's bias (as discussed above), subjectivity and rigor (Smith et al., 2009). However in this study, through reflection and supervision the researcher made efforts to ensure quality was establish by gaining a good understanding of IPA.

The sample recruited for this study was small and from a specific geographical region (London). As a result, the findings from this study cannot be generalised as results may not be typical of older Black Caribbean adults living outside London. Nonetheless, the study's small sample size may not necessarily be considered as a disadvantage, as it allowed for more rich and meaningful data. Furthermore, IPA allows for a smaller sample size as it entails having an idiographic approach, whereby the group selected is broadly homogenous, concerned with understanding a particular phenomenon or context (Smith \& Osborn, 2003).

The Caribbean consists of a number of is lands; as a result, help seeking views towards mental health may differ among the various is lands. This study is not representative of each Caribbean island, which may in turn be seen as a limitation. What is more, it may be possible that other older Black Caribbean adults who did not take part in the current study regardless of geographical area may have very different experiences of help seeking or coping with depression which has not been found in this study.

Nevertheless, as discussed in Chapter 3the researcher when considering the sampling criteria ensured selection was theoretically congruent with the study's paradigm and framework for analysis. For this reasons, in this study participants were selected purposively instead of through probability approaches (Smith et al., 2009; Chapman and Smith, 2002).Furthermore, participants were selected to enable the researcher to access views on the subject under investigation; they symbolise a point of view, rather than people on the whole.

To conclude this section, participants in this study were particularly motivated to take part because they either had a relative or friend with mental health problems; the subject 
matter related to their occupation prior retirement or due to the ir role in the community. For these reasons, there may be potential bias in the research; that is, it may possible that participants' perception of mental health services and help seeking may be heavily influenced from having close contact with somebody who has already had access to wellbeing and/or mental health services. Nonetheless, the aims of this study are to represent a particular perspective.

\subsection{Conclusions and Recommendations for Future Research}

The findings in this study give some light into the experiences of a sample of the UK's older Black Caribbean adults on coping with depression and the ir help seeking views in relation to this phenomenon. The findings from this study are consistent with some findings from previous studies; for example, highlighted were common help seeking attitudes held within the Black Caribbean population such as" just get on with it". In addition, the study unveiled various subjective components to coping with depression; in particular, the findings showed how participants past personal experiences of coping with depression, including migratory histories, cultural and religious views, and personal relations hips influenced the ir help seeking views and preferred coping methods for depression.

The results from this study are also relevant to Counselling Psychology. The knowledge gained is not only valuable for future work with this client group; but is also consistent with the values of Counselling Psychology which is to empower individual's steps in self-development, by working with the ir unique subjective or inter-subjective psychological experience. In addition, the results from this study are also in line with guidance from the Department of Health, which advocates service users' views and experiences as an effective and crucial part of evaluating health services - including mental health services (NICE, 2013).

The study could contribute to future research in this area. For example, further study could explore the perspectives of the Black Caribbean community who have recently migrated to the UK. This would allow for a wider and more comprehensive picture of how Black Caribbean people comprehend looking for help for mental health difficulties. It may also be interesting to compare the difference between British born Black Caribbean people and 1st generation Black Caribbean peoples' help seeking views towards mental health service use. 
In addition, in order to better understand help seeking, as well as the community context of the UK's Black Caribbean community, future studies could also explore further the aspects which enable help seeking within the community. For example, research could explore the community support already in place (i.e. Afro Caribbean mental health projects for older adults); as well as explore how mental health is discussed in this context. It may also be interesting to explore how mental health is discussed within the families of Black Caribbean people, as the findings from this study has shown the significant impact of " upbringing" on help seeking views/attitudes.

Given that the sample in this study was not representative of each Caribbean Island, it may be interesting to do a comparative study on the help seeking views towards mental health service use across the various Caribbean Islands. The area known as the Caribbean is a group of over 7000 is lands which comprises of 13 sovere ign states and 2 dependent territories and is located by the Caribbean Sea (Caribbean islands, 2020). Given the high number of Caribbean islands, research could focus on the islands that have a longstanding history of migration to the UK. The learning gained may be useful to improve service provision and promote the integration of mental health services. In addition, further to promoting the integration of mental health services, future studies could also do a comparative study on help seeking views across the genders among older Black Caribbean adults.

On the whole, there is already a focus in the UK on increasing access to mental health service use among the Black and ethnic minorities; nevertheless, although the need for accessible services is widely recognised, it is important for mental health services to not only be accessible, but they must also be suitable and pertinent to the populations they serve. For these reasons, future research could explore further the current models of practice in mental health services. It is known that the models used in mainstream services are underpinned by Eurocentric ideas; as a result, it may useful to do a comparative study on alternative therapeutic approaches which incorporate social and cultural factors on mental health. 


\section{REFERENCES}

Age UK Buckinghamshire.(2016). No One Should Have No One.Available at www.ageuk.or.uk/buckinghams hire/newsandcampaigns 1/nooneshouldhavenoe, accessed on 6 November 2016

Age concern and the Mental the Mental Health Foundation.(2006).Promoting Mental health and Wellbeing in later life. A first report from the UK Inquiry into Mental Health and Wellbeing in later life. London: Age Concern and Mental Health Foundation. Available at www.mentalhealth.org.uk/sites/default/files/promoting mh wb later life .pdf, accessed on 6 November 2016

Asnaani, A., \& Hofmann, S.G. (2012). Collaboration in multicultural therapy: Establishing strong therapeutic alliance across cultural lines. Journal of Clinical Psychology, 68,187-197.

Avenier, M. J., \& Thomas, C. (2015). Finding one's way around various methodological guidelines for doing rigorous case studies: A comparison of four epistemological frameworks. Systèmesd information \& management,20(1), 61-98.

Baldw in, R. C. (2014). Depression in later life (2 ${ }^{\text {nd }}$ edition): Oxford psychiatry library. Oxford. Oxford University Press.

Banister, P. B., Parker, E., \& Taylor, I. M. and Tindall, C. (1994)."Qualitative methods in psychology: A research guide. Buckingham. Open University Press

Bentall, D. P. R. (1999). The medicalisation of misery: A critical realist analysis of the concept of depression. Journal of mental health, 8(3), 261-274.

Bhui, K. S. (2013). Public mental health and inequalities. Elements of culture and mental health. Critical questions for clinicians, 73-75.

Bhui, K., \&Bhugra, D. (2002). Mental illness in Black and Asian ethnic minorities: Pathways to care and outcomes. Advances in Psychiatric Treatment, 8(1), 26-33.

Bhugra, D., \& Gupta, S. (Eds.). (2011). Migration and mental health.Cambridge. Cambridge University Press. 
Bhugra, D., Harding, C., \& Lippett, R. (2004).Pathways into care and satisfaction with primary care for black patients in South London.Journal of Mental health, 13(2), 171183.

Borowsky, S. J., Rubenstein, L. V., Meredith, L. S., Camp, P., Jackson-Triche, M., \& Wells, K. B. (2000). Who is at risk of non-detection of mental health problems in primary care?.Journal of general internal medicine, 15(6), 381-388.

Bowes, A., \& Wilkinson, H. (2003). 'We didn't know it would get that bad': South Asian experiences of dementia and the service response. Health \& social care in the community, 11(5), 387-396.

BPS Division of Counselling Psychology.

(2014).http://www.bps.org.uk/sites/default/files/documents/professional practice guide lines -division of counselling psychology.pdf,accessed 1st October 2014

British Psychological Society (2020) https://thepsychologist.bps.org.uk/volume33/january-2020/bame-representation-and-psychology, accessed 12 December 2019

British Psychological Society (2018) Guidance for Psychologists on working with Community Organisations

www.bps.org.uk/sites/www.bps.org.uk/files/Policy/Policy\%20\%20Files/Guidance $\% 20$ for $\% 20$ psychologists $\% 20$ on $\% 20$ w orking $\% 20 \mathrm{with} \% 20 \mathrm{co}$ mmunity\%20organisations\%20(Sep\%202018).PDF , accessed 02 April 2020

Brown, J.S.L., Boardman, J., Whittinger, N., Ashworth, M. (2010). Can a self-referral system improve access to psychological treatments? British Journal of General Practice $60,365-371$

Carr, S. (2014). Social Care for Marginalised Communities: Balancing selforganisation, micro-provision and mainstream support. Health Services Management.

Caribbean Islands (2020) https://www.caribbeanis lands.com/ accessed 12.4.2020

Centre for policy on ageing (2014).Resilience in Older age. Available at www.cpa.org.uk/information/reviews/CPA-Rapid-Review-Resilience-and-recovery.pdf, accessed on 6 November 2016 
Chapman, E., \& Smith, J. A. (2002).Interpretative phenomenological analys is and the new genetics.Journal of health psychology, 7(2), 125-130.

Charmaz, K. C. (2006). Grounded Theory. In J. A. Smith (Ed.), Qualitative Psychology: A practical guide to research methods (pp. 81-110). London. Sage.

Cinnieralla, K and Lowenthal, K.M .(1999). Religious and ethnic group influences on beliefs about mental illness: A qualitative interview study. Bristish Journal of Medical Psychology 72, 2, 505-524.

Conner, K. O., Copeland, V. C., Grote, N. K., Rosen, D., Albert, S., McMurray, M. L. \&Koeske, G. (2010).Barriers to treatment and culturally endorsed coping strategies among depressed African-American older adults.Aging and Mental Health, 14(8), 971983.

Cornwe1l, E. Y., \& Waite, L. J. (2009).Social disconnectedness, perceived isolation, and health among older adults.Journal of health and social behaviour, 50(1), 31-48.

Corrigan, P. (2004). How stigma interferes with mental health care. American psychologist, 59(7), 614.

Creswell, J. W. (2013). Research design: Qualitative, quantitative, and mixed methods approaches. Sage publications.

Dein, S., Persaud, A., Tribe, R.,Lashley, M., Bhui, K., Thapliyal, A., Khatib, Y., Rathod, S., Gabriel, M., Willis, J., Bhugra, D., Rathod, S., . Braakman, M.H., Iraqi ,F., Swartz, L., Jisraj Singh Gataora: Van Sluytman, L.G., Bass, D., Bemak:, F., Dinos,S., Day, G., Watson,S., ChiYing Chung, R.(2019) Moving Beyond Christianity: Islam, Judaism, Hinduism and Mental Health: A Centre for Applied Research and Evaluation International Federation (Careif) Global Position statement.World Association of Cultural Psychiatry Research Review Journal 14 1/2, $13-19$

Department of Health (2014), https://www.gov.uk/government/policies/making-mental$\underline{\text { health-services-more-effective-and-accessible--2 }}$ accessed $1^{\text {st }}$ October 2014

Department of Health (2005).Delivering Race Equality in Mental Health Care: An action plan for reform inside and outside services and the Government's response to the 
Independent inquiry into the death of David Bennett.

http:/webarchive.nationa larchives.gov.uk/20130107105354/http:/www.dh.gov.uk/en/pu blicationsandstatistics/publications/publicationspolicyandguidance/dh 4100773 accessed 12 December 2019

Fade, S. (2004). Using interpretative phenomenological analys is for public health nutrition and dietetic research: a practical guide.Proceedings of the nutrition society, 63(4), 647-653.

Fassil, Y., \& Burnett, A. (2014).Commissioning mental health services for vulnerable adult migrants.Guidance for commissioners.

Fernando, S. (2004). Cultural diversity, mental health and psychiatry: The struggle against racism. Routledge.

Fernando, S. (2014). Mental health worldwide: Culture, globalization and development. Springer.

Finlay, L., \& Gough, B. (2003).Introduc ing reflexivity.Reflexivity: A practical guide for researchers in health and social sciences, 1-2.

Forbes, L. J. L., Simon, A. E., Warburton, F., Boniface, D., Brain, K. E., Dessaix, A.\& Lockwood, G. (2013). Differences in cancer awareness and beliefs between Australia, Canada, Denmark, Norway, Sweden and the UK (the International Cancer Benchmarking Partnership): do they contribute to differences in cancer survival?.British journal of cancer, 108(2), 292.

Furnham, A., \&Chan, E. (2004).Lay theories of schizophrenia: A cross-cultural comparison of British and Hong Kong Chinese attitudes, attributions and beliefs. Social Psychiatry and Psychiatric Epidemiology, 39, 543-552.

Goldberg, D., \& Huxley, P. (1980). Mental illness in the community: the pathway to psychiatric care London. New York, Tavistock.

Gooding, P. A., A. Hurst, J. Johnson, and N. Tarrier."Psychological resilience in young and older adults."International journal of geriatric psychiatry 27, no. 3 (2012): 262270. 
Goudie, F. (2003).Psychological Therapy with Older adults. In R. Woolfe, W. Dryden and S. Strawbridge (eds) Handbook of Counselling Psychology. London: Sage

Guest, G., Bunce, A., \& Johns on, L. (2006). How many interviews are enough? An experiment with data saturation and variability.Field methods, 18(1), 59-82.

Gulliver, A., Griffiths, K. M., Christensen, H., \& Brewer, J. L. (2012).A systematic review of help-seeking interventions for depression, anxiety and general psychological distress.BMC psychiatry, 12(1), 81 .

Gum, A. M., Areán, P. A., Hunkeler, E., Tang, L., Katon, W., Hitchcock, P. \&Unützer, J. (2006). Depression treatment preferences in older primary care patients. The Gerontologist, 46(1), 14-22.

Harper, D. J. (2013). On the persistence of psychiatric diagnosis: Moving beyond a zombie classification system. Feminism \& Psychology, 23(1), 78-85.

Hays, P. (2001).Addressing cultural complexities in practice: A framework for clinicians and counsellors. Washington: American Psychological Association

Hays, P \& Iwamasa, G.Y. (2006). Culturally responsive cognitive-behavioural therapy: Assessment, practice and supervision. Washington: American Psychology Association

Hays, D. G., \& Wood, C. (2011).Infusing qualitative traditions in counseling research designs.Journal of Counseling \& Development, 89(3), 288-295.

Huang, Y., \& Carpenter, I. (2011).Identifying elderly depression using the Depression Rating Scale as part of comprehensive standardised care assessment in nursing homes.Aging \& mental health, 15(8), 1045-1051.

Huq, A., McIntosh, M. \& Tribe, R. (2020) Professional and Ethical Issues in Working with Older Adults in Tribe, R \& Morrissey, J. (eds) (2020) The Handbook of Professional, Ethical and Research Practice for Psychologists, Psychotherapists, Counsellors, Psychiatrists and Social Workers, London: Brunner- Routledge. 3rd edition, 197-207

Improving Acess to Psychological Therapies.(IAPT).(2009) Older People-Positive Practice Guide. guide.pdf, accessed on 12December 2019 
Jacob, K. S., Bhugra, D., Lloyd, K. R., \& Mann, A. H. (1998).Common mental disorders, explanatory models and consultation behaviour among Indian women living in the UK.Journal of the Royal Society of Medicine, 91(2), 66-71.

Kamiya, Y., Doyle, M., Henretta, J. C., \& Timonen, V. (2013). Depressive symptoms among older adults: The impact of early and later life circumstances and marital status. Aging \& mental health, 17(3), 349-357.

Kaplan, D. B., \& Berkman, B. J. (2016). Effects of life transitions on the elderly.Consumer Version.Prieiga per interneta: $<$ https://www. merckmanuals. $\mathrm{com} / \mathrm{professional/geriatrics/socialissues-in-the-elderly/effects-of-life-transitions-on-the-}$ elderly.

Karademas, E. C. (2009). Counse lling psychology in medical settings: The promising role of counselling health psychology.The European Journal of Counselling Psychology, 1(1/2).

Kasket, E. (2012). The counselling psychologist researcher. Counselling Psychology Review, 27(2), 64-73.

Lane, P. (2017).Conceptualising Aging and Anti-discriminatory Practice. In: Lane, P., and Tribe, R., ed.,Anti-Discriminatory Practice in Mental Health Care for Older People. Jessica Kingsley Publishers, pp.19-47

Larkin, M., Watts, S., \& Clifton, E. (2006). Giving voice and making sense in interpretative phenomenological analysis. Qualitative research in psychology,3(2), 102-120.

Lawrence, V., Banerjee, S., Bhugra, D., Sangha, K., Turner, S., \& Murray, J. (2006). Coping with depression in later life: a qualitative study of help-seeking in three ethnic groups.Psychological medicine, 36(10), 1375-1383

Lazarus, R. S., \& Folkman, S. (1984). Coping and adaptation. The handbook of behavioural medicine, 282325.

Magnani, R., Sabin, K., Saidel, T., \& Heckathorn, D. (2005). Review of sampling hardto-reach and hidden populations for HIV surveillance. Aids, 19, 67-72. 
McIntosh, M and Huq, A.(2017).Common Mental Health Problems. In: Lane, P., and Tribe, R., ed.,Anti-Discriminatory Practice in Mental Health Care for Older People. Jessica Kingsley Publishers, pp.102-121

Mackenzie, C. S., Gekoski, W. L., \& Knox, V. J. (2006). Age, gender, and the underutilization of mental health services: the influence of help-seeking attitudes. Aging and Mental Health, 10(6), 574-582.

Macran, S., \& Shapiro, D. A. (1998). The role of personal therapy for therapists: A review. British Journal of Medical Psychology, 71(1), 13-25.

Madill, A., Jordan, A. \& Shirley, C. (2000). Objectivity and reliability in qualitative analysis: Realist, contextualist and radical constructionist epistemologies. British Journal of Psychology, 91, 1-20.

Maxie, A.C., Arnold, D.H. \& Stephenson, M .(2006). Do therapists address ethnic and racial differences in cross-cultural psychotherapy. Psychotherapy: Theory, Research, Practice, Training, 43, 85-98

McLeod, J. (2003). Qualitative research methods in counselling psychology. In: R. Woolfe, W. Dryden and S. Strawbridge, ed., Handbook of Counselling Psychology, 2nd ed. London: SAGE Publications, pp.74-92.

Mental Health Foundation.(2019).https://www.mentalhealth.org.uk/a-to-z/b/blackasian-and-minority-ethnic-bame-communities, accessed 02 April 2020

Mental Health Foundation.(2016). Fundamental Facts about Mental

Health.https://www.mentalhealth.org.uk/publications/fundamental-facts-about-mental-

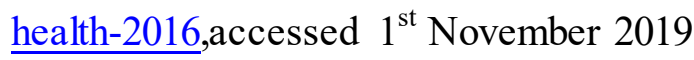

Mental Health Foundation.(2007). Fundamental

Facts.http://www.mentalhealth.org.uk/content/assets/PDF/publications/fundamental_fa cts_2007.pdf?view=Standard, accessed 1 st October 2014

Mental Health and Older People (2013).Retrieved $1^{\text {st }}$ October 2014, fromhttp:/Wfmh.com/wp-content/uploads/2013/11/2013_wmhday_english.pdf 
Mertens, D.M. (2005). Research methods in education and psychology: Integrating diversity with quantitative and qualitative approaches. (2nd ed.) Thousand Oaks: Sage.

Miller, T., Velleman, R., Rigby, K., Orford, J., Tod, A., Copello, A., \& Bennett, G.(1997). The use of vignettes in the analys is of interview data: Relatives of peoplew ith drug problems. In N. Hayes (Ed.), Doing qualitative analysis in psychology(pp. 201225). Hove: Psychology Press.

Mind .(2013). Mental Health in Crisis Care:Commissioning Excellence for Black and Minority Ethnic Groups. Stratford:Mind

Morgan, R., Khan, A., McFarlane, F., Thomas, L. \& Ram du Sautoy, S. (2009). Access to talking therapies: The views and experiences of people from Black and minority ethnic communities in secondary care in East London. Clinical Psychology Forum 196, $37-40$.

Mortimer, J., \& Green, M. (2015). Briefing: the health and care of older people in England 2015. Age UK, London. http://www. ageuk. org. uk/professional-resourceshome/research/reports/care-and-support/the-health-and-care-of-older-people-inengland-2015/(accessed 22 September 2016).

Mulvaney-Day, N. E., Earl, T. R., Diaz-Linhart, Y., \&Alegría, M. (2011). Preferences for relational style with mental health clinicians: A qualitative comparis on of African American, Latino and Non-Latino White patients. Journal of clinical psychology, 67(1), $31-44$.

National Institute for Health and Social Care (NICE) (2016) NG 44 on Community engagement: improving health and wellbeing and reducing health inequalities. https://www.nice.org.uk/guidance/ng44 accessed 22.04.2020

National Institute for Health and Clinical Excellence. (NICE). (2013). Patient and public involvement policy, https://www.nice.org.uk/media/default/About/NICECommunities/Public-involvement/Patient-and-public-involvement-policy/Patient-andpublic-involvement-policy-November-2013.pdf, accessed 12 December 2019 
National Institute for Health and Clinical Excellence. (NICE), (2011). Service user experience in adult mental health: improving the experience of care for people using adult NHS mental health services, https://www.nice.org.uk/guidance/cg136, accessed 12 December 2019

NIMHE, (2003).Inside Outside: Improving Mental Health Services for Black and Minority Ethnic Communities in England p10, National Institute for Mental Health in England, Leeds

NHS England .(2014.) A Call to Action: Achieving Parity of Esteem Transformation Ideas for Commissioners. Available at www.england.nhs.uk/wpcontent/uploads/2014/02/nhs. parity.pdf, accessed on 6 November 2016

National Health Service Mental Health.(1999). National Service Frameworks London: NHS p77,

Office for National Statistics.(ONS). (2019). Overview of the UK population: August 2019.

Office of National Statistics.(ONS).(2018). Ethnicity and national identity in England and Wales 2018.

O'Leary, Z. (2004). The essential guide to doing research. London: Sage.

Patel, N., Bennett, E., Dennis, M., Dosanjh, N., Miller, A., Mahtani, A. \& Nadirshaw, Z. (eds). (2000). Clinical Psychology, 'Race \& Culture: A Training Manual. Blackwell: Chichester.

Pietkiewicz, I., \& Smith, J. A. (2014). A practical guide to using Interpretative Phenomenological Analysis in qualitative research psychology.Psychological Journal, 20(1), 7-14

Ponterotto, J. G. (2005). Qualitative Research in Counseling Psychology: A Primer on Research Paradigms and Philosophy of Science. Journal of Counseling Psychology, 52(2), 126-136. 
RCP. (2009). Age Discrimination in Mental Health Services:Making Equality a Reality. Position Statement PS2/2009. London:RCP. Available at www.rcpsych.ac.uk/pdf/PS02 2009x.pdf,accessed on 20 September 2016

Reid, K., Flowers, P. and Larkin, M. (2005). Exploring lived experience. The Psychologist, 18(1), pp.20-23.

Rennie, D. L. (2007).Methodical hermeneutics and humanistic psychology.The Humanistic Psychologist, 35(1), 1-14.

Rickwood D, Braithwaite V: Social-psychological factors affecting help-seeking for emotional problems. Social science \& medicine (1982). 1994, 39 (4): 563-572.

Rickwood D, Deane F, Wils on C: When and how do young people seek professional help for mental health problems?. Med J Aust. 2007, 187 (7): S35-39.

Sadler G., Lee H., Lim R., \& Fullerton J. (2010).Recruitment of hard-to-reach population subgroups via adaptations of the snowball sampling strategy. Nursing \& Health Issues, 12, 369-374.

Sainsbury Centre for Mental Health. (2002). Breaking the circles of fear: A review of the relationship between mental health services and African and Caribbean communities. Sainsbury Centre for Mental Health.

Shah, A. (2008).Estimating the absolute number of cases of dementia and depression in the black and minority ethnic elderly population in the United Kingdom.International Journal of Migration, Health and Social Care, 4(2), 4-15.

Smith, J. ,Flowers, P. \& Larkin, M. (2009). Interpretative Phenomenological Analys is:T heory, Method and Research. London: Sage

Stevenson, J., and Rao, M. (2014). Explaining levels of Wellbeing in BME populations in England. African Health Policy Network.

The king's Fund .https://www.kingsfund.org.uk/publications/social-prescribing, accessed 12 December 2019 
Tribe, R. (2017).Ageing, Ethnicity and Mental health.In: Lane, P., and Tribe, R., ed.,Anti-Discriminatory Practice in Mental Health Care for Older People. Jessica Kingsley Publishers, pp.69-101

Tribe , R. (2014) Culture, politics and global mental health. Disability and the Global South 1,2, 251-265.

Tribe, R. \& Bell, D. (2018) Social justice, diversity and leadership: How counselling psychologists can get involved in work which promotes leadership and diversity beyond the consulting room. European Journal of Counselling Psychology,6,1,111-125

Tribe, R., \& Morrissey, J. (Eds.).(2015). Handbook of professional and ethical practice for psychologists, counsellors and psychotherapists.Routledge.

Vanleerberghe, P., De Witte, N., Claes, C., Schalock, R. L., \& Verté, D. (2017). The quality of life of older people aging in place: a literature review. Quality of Life Research, 26(11), 2899-2907.

Watts, S. C., Bhutani, G. E., Stout, I. H., Ducker, G. M., Cleator, P. J., McGarry, J., \& Day, M. (2002). Mental health in older adult recipients of primary care services: is depression the key issue? Identification, treatment and the general practitioner.International Journal of Geriatric Psychiatry, 17(5), 427-437.

Willig, C. (2008). Introducing qualitative methods in psychology. Buckingham: Open University Press.

Willig, C., \&Stainton-Rogers, W. (Eds.).(2008). The SAGE handbook of qualitative research in psychology. Sage

Williams, E. D., Tillin, T., Richards, M., Tuson, C., Chaturvedi, N., Hughes, A. D., \& Stewart, R. (2015). Depressive symptoms are doubled in older British South Asian and Black Caribbean people compared with Europeans: associations with excess comorbidity and socioeconomic disadvantage. Psychologicalmedicine, 45(9), 1861-1871.

Wills, C. E., \& Holmes-Rovner, M. (2006). Integrating decision making and mental health interventions research: research directions. Clinical Psychology: Science and Practice, 13(1), 9-25. 
Woodward, A. T., Taylor, R. J., Abelson, J. M., \& Matusko, N. (2013). Major depressive disorder among older African Americans, Caribbean blacks, and nonHispanic whites: secondary analys is of the national survey of American life. Depression and anxiety, 30(6), 589-597.

World Health Organisation. (WHO) (2020) Community engagement for quality, integrated, people-centred and resilient health

serviceswww.who.int/servicedeliverysafety/areas/qhc/community-engagement/en/, accessed 02 April 2020

World Health organisation.(WHO). (2016). Mental Health and Older Adults FACT Sheet www.whp.int/medicawntre/factsheets, accessed 02 April 2020

World Population Prospects.(2019): Highlights. New York (US): United Nations Department for Economic and Social Affairs.

Yardley, L. (2000). Dilemmas in qualitative health research. Psychology and Health, [online] 15(2), pp.215-228. Available at: http://tandfonline.com, accessed $1^{\text {st }}$ October 2014.

Yardley, L. (2008). Demonstrating validity in qualitative psychology. In: J. Smith, ed., Qualitative Psychology: A Practical Guide to Research Methods, 2nd ed. London: SAGE Publications, pp.235-251. 


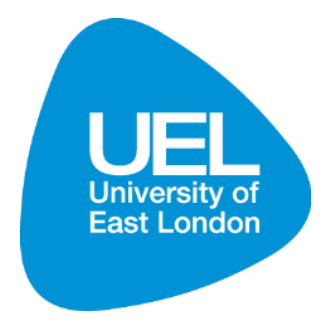

\section{UNIVERSITY OF EAST LONDON}

The following questions will be used as a guide with the general addition of prompts (for example, could you tell me more about that? How did you feel about that? What did that mean for you? What was that like? etc)

1. Can you tell me what your understanding of Depression is?

Further explorative prompts: Can you tell me what behaviours, thoughts or feelings tell you someone is depressed?, What type of situations do feel can make someone depressed

2. Thinking about the behaviours thoughts or feelings of someone who is depressed can you tell me about any experiences, if any, where you felt depressed?

Further explorative prompts: why do you think you felt depressed at that time of your life? What was going on in your mind then? (Thoughts/ associations/ fantasies) how long did this experience last/ episode last? How did you interpret the behaviours/ thoughts feelings? How did it feel go through that process? What kind of help was available (medical or non medical)? What was helpful or unhelpful about the help available? What helped you to cope? What made it worse?

3. Can you tell me of your experiences if any with someone within the Black Caribbean community who is depressed?

Further explorative prompts: why do you think they felt depressed at that time of their life? What was going on in your mind then? (Thoughts/ associations/ fantasies) how long did this experience last? How did you interpret the behaviours/ thoughts feelings? How did it feel go through that process? What kind of help was available (medical or non medical)? What was helpful or unhelpful about the help available? What helped them to cope? What made it 
worse?

4. Can you tell me of your experiences if any with older adults or Peers with the Black Caribbean community in the UK who are depressed?

Further explorative prompts: Why do you think they felt depressed at that time of their life? What was going on in your mind then? (Thoughts/ associations/ fantasies) how long did this experience last? How did you interpret the behaviours/ thoughts feelings? How did it feel go through that process? What kind of help was available (medical or non medical)? What was helpful or unhelpful about the help available? What helped them to cope? What made it worse?

\section{What do you think could help someone feel better if they were depression?}

Further explorative Prompts: Can you tell me about any treatments available for people who are depressed (Medical/non-medical) If not- why? What kind of treatment do you think is most appropriate? Why? What do you think should have been done to help you or others (me dical / non-medical treatment)?

\section{What decisions will someone have to make when seeking help with depression?}

Further explorative prompts: How did you or others decide to go about seeking help with depression? Where do you seek help? What stopped you from seeking help? Do you know where to seek help? If sought medical / nonmedical treatment: What did you think about recommendations you received? What was helpful or unhelpful about the recommendations received? What was similar or different to what you thought about depression? What thoughts / feelings do you have about the referral process? What were your thoughts/ feelings towards the person/s that completed any assessments? 
APPENDIX B - Information Sheet

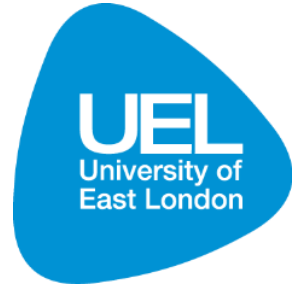

\section{UNIVERSITY OF EASTLONDON}

\section{The Principal Investigator}

Natalie Bailey

Email: u1137793@uel.ac.uk

\section{Consent to Participate in a Research Study}

The purpose of this letter is to provide you with the information that you need to consider in deciding whether to participate in the research study. The study is being conducted as part of my Professional Doctorate of Counselling Psychology degree at the University of East London.

\section{Project Title}

Coping with depression in later life: A qualitative study of help-seeking attitudes among older Black Caribbean adults in the UK.

\section{Project Description}

Mental health providers need to comprehend the decisions and experiences (of service users) that influence help seeking behaviour to deliver effective, culturally appropriate and accessible services. However, the influencing factors of help seeking behaviour are not often explored within older adults from ethnic minority groups. For these reasons, this study proposes to explore the UK's older Black Caribbean adults' experiences of coping with depression in later life - Specifically exploring their help seeking attitudes, perceptions and beliefs about what will help someone cope with depression. Gaining further knowledge on the experiences of older Black Caribbean people could potentially aid Counselling Psychologists in developing appropriate helpseeking strategies which can be defined culturally to meet the needs of older people from Black Caribbean origin in the UK.

The study will include Black Caribbean Men and women aged 65 or over. To participate in the interview, you do not need to be clinically depressed as the primary focus of this study is to explore help seeking views/attitudes within the general public instead of service users who are prescribed medication from their GP/or secondary care, to cope with low mood; or have an active referral to psychiatric care. However, participants will have some personal experience of self-defined depression across their lifespan.

As part of the research, you will be asked to participate in an interview which will last approximately 50 minutes to 1 hour although the length of time will vary depending on how much information you feel comfortable to share. The questions will generally focus on your understanding of coping with depression, including your beliefs and expectations with what will help someone with depression. The interviews will be audiorecorded, 
Some people feel that talking about their experiences openly can be quite therapeutic although personal information can sometimes bring up difficult feelings. As a participant, it is within your right to not answer questions that make you feel uncomfortable or distressing. You can rest assured that you can withdraw from the study at any time. You will also be provided with a list of contact information if you feel you need further support.

Once the transcribed data has been analysed, you will have the opportunity to read the data and make any further comments you wish to add.

\section{Confidentiality of the Data}

Everything you share will remain confidential. All your personal details will only be accessible to the researcher. The recorded interview will be transcribed and your personal details will be anonymised in the transcript. All audio recordings will be erased once the research is complete. Transcribed information will be kept up to 3 years and will be stored securely.

\section{Location}

If you agree to take part in the research, an interview date, time, and location will be arranged that is suitable for you. The interview is likely to take place in a private room at your day centre or lunch club.

\section{Disclaimer}

You are not obliged to take part in this study and should not feel coerced. You are free to withdraw at any time. Should you choose to withdraw from the study you may so without disadvantage to yourself and without any obligation to give a reason. If you do withdraw from the research, all your personal details and interview material will be erased and will not be used for the research write-up.

Please feel free to ask me any questions. If you are happy to continue you will be asked to sign a consent form prior to your participation. Please retain this in vitation letter for reference.

If you have any questions or concerns about how the study has been conducted, please contact the study's supervisors:

Professor Rachel Tribe (r.tribe@uel .ac.uk) or Dr Libby Watson (I.watson@uel.ac.uk)

School of Psychology, University of East London, Water Lane, London E15 4LZ.

\section{$\underline{\text { OR }}$}

Chair of the School of Psychology Research Ethics Sub-committee: Dr. Mark Finn, School of Psychology, University of East London, Water Lane, London E15 4LZ.(Tel: 0208223 4493. Email: m.finn@uel.ac.uk)

Thank you in anticipation.

Yours sincerely,

Natalie Bailey 
APPENDIX Ba - Email Recruitment Example 1

From: Natalie Bailey

Sent: 01 October 2016 11:24

To: Rev John Macauley

Subject: Coping with depression among Black Caribbean Older Adults

Hello Reverend John

I hope you are well.

Please see attached my Doctoral Research advert/ information sheet and circulate as and where for you feel necessary. Perhaps you might know someone who can help?. Urgently in need for participants.

Thanking you in advance.

I look forward to your response.

Warmest regards,

Natalie Bailey 
Coping with depression in Later Life among Black Caribbean Older Adults

\section{APPENDIX Ba - Email Recruitment Example 2}

From:

Sent: 06 October $201616: 33$

To:

Subject: Re: Coping with depression among Black Caribbean Older Adults

ok and blessings Natalie

Reverend John B. Macaulay,M.Soc. Sc.

Minister

Upper Clapton United Reformed Church

85a Upper Clapton Road

London, E5 $98 \mathrm{BU}$

Tel: tomen

Mobile:

Email:

In a message dated 05/10/2016 14:15:51 GMT Daylight Time,

Thank you for taking the time to go through this.

Your guidance is much appreciated.

I look forward to our next catch up re this matter.

Warmest regards

Natalie

Get Qutlook for Android

On Wed, Oct 5, 2016 at 3:53 AM +0700,

Hello Natalie

I have read your email. It is very interesting and needed. I will have a chat with you regarding names I will suggest.

Blessings

Reverend John B. Macaulay,M.Soc. Sc.

Minister

Upper Clapton United Reformed Church

85a Upper Clapton Road

London, E5 98U

Tel: ganonenes.

Mobile:

Email:

In a message dated 01/10/2016 14:53:26 GMT Daylight Time,

writes: 


\section{APPENDIX Ba - Email Recruitment Example 3}

Coping with depression in Later Life among Black Caribbean Older Adults

Natalie Bailey

To: Natalie Bailey

From:

Sent: 13 June 2016 16:42

To: Natalie Bailey

Subject: Re: Coping with depression in Later Life among Black Caribbean Older Adults

Dear Natalie,

Would be happy to meet it would cost $u$ a mocha coffee, and I would be happy to give u my insights, Txt me to arrange a

meet.

Kind Regards

Sent: Monday, June 13, 2016 at 1:15 PM

From: "Natalie Bailey"

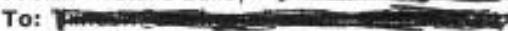

Cc: "Natalie UEL"

Subject: Coping with depression in Later Life among Black Caribbean Older Adults

Dear

I hope you are well.

It was lovely speaking with you the other day, although brief it was quite insightful.

Thank you for agreeing to review my project. As discussed, please find attached the information sheet/advert for my research.

I was hoping we could meet to complete a pilot interview as well as discuss possible future connections for the project.

I hope this is OK?

1 look forward to hearing from you soon.

Kind regards,

Natalie Bailey 


\section{APPENDIX Ba - Email Recruitment Example 4}

Coping with depression in Later Life among Black Caribbean Older Adults

Natalie Bailey

To: Natalie Bailey

6) 1 attachments $(26 \mathrm{~K} 6)$

Natalie Bailey Project Description Letter.dooc

From: Natalie Bailey

Sent: 01 October 2016 18:14

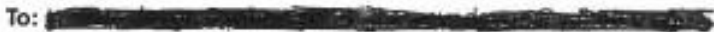

Subject: Coping with depression among Black Caribbean Older Adults

Dear Dr

I hope you are well.

Please see attached my doctoral research advert/information sheet and circulate as and where for you feel necessary. Perhaps you might know someone who can help? Urgently in need for participants.

Thanking you in advance.

I look forward to your response.

Kind regards,

Natalie 


\section{UNIVERSITY OF EAST LONDON}

\section{Consent to participate in a research study}

Coping with depression in later life: A qualitative study of help-seeking attitudes amongOlder Black Caribbean adults in the UK.

I have read the information sheet relating to the above research study and have been given a copy to keep. The nature and purposes of the research have been explained to me, and I have had the opportunity to discuss the details and ask questions about this information. I understand what is being proposed and the procedures in which I will be involved have been explained to me.

I understand that the interview will be audio recorded and the data will be anonymised. I understand that my involvement in this study, and particular data from this research, will remain strictly confidential. Only the researcher involved in the study will have access to identifying data. It has been explained to me what will happen once the research study has been completed.

I hereby freely and fully consent to participate in the study which has been fully explained to me. Having given this consent I understand that I have the right to withdraw from the study at any time without disadvantage to myself and without being obliged to give any reason.

Participant's Name (BLOCK CAPITALS)

Participant's Signature

Researcher's Name (BLOCK CAPITALS)

Researcher's Signature

Date: 


\section{UNIVERSITY OF EAST LONDON}

\section{Next Steps}

Thank you for taking part in this study, your contributions are invaluable and are much appreciated. Once the transcribed data has been analysed, you will have the opportunity to read the data and make any further comments you wish to add.

Everything you share will remain confidential. All your personal details will only be accessible to the researcher. Your personal details will be anonymised in the transcript and all audio recordings will be erased once the research is complete. Transcribed information will be kept up to 3 years and will be stored securely.

\section{Further support for future reference}

Some people feel that talking about their experiences openly can be quite therapeutic although personal information can sometimes bring up difficult feelings. As a result, if at any time you feel that your mood has worsened significantly, please contact your GP.

In addition, you may also find it helpful to contact other sources of support when feeling distressed, such as

- The Samaritans, available 24 hours a day, on 08457909090 (or via text: 07725 909090)

- SaneLine, available from 6pm-11pm 365 days a year, on 08457678000

- SupportLine, available for about 2 hours, Monday-Thursday evenings, on 01708 765200

- Crisis Line, 0300456 8342, for professional help

- NHS 111 (if you feel you urgently need medical help or advice but it's not a lifethreatening situation)

- Attend A\&E department

- Age UK advice: 08001692081

It was a pleasure to work with you; I send you warm good wishes for the future,

Yours sincerely

Natalie Bailey 


\section{School of Psychology Research Ethics Committee}

\section{NOTICE OF ETHICS REVIEW DECISION}

For research involving human participants

$\mathrm{BSc} / \mathrm{MSc} / \mathrm{MA} /$ Professional Doctorates in Clinical, Counselling and Educational

Psychology

SUPERVISOR: Rachel Tribe

STUDENT:Natalie Victoria Bailey
REVIEWER: Matteo Martini

Title of proposed study: Coping with depression in later life: A qualitative study of helpseeking attitudes among Older Black Caribbean adults in the UK.

Course: Professional Doctorate in Counselling Psychology

\section{DECISION OPTIONS:}

1. APPROVED: Ethics approval for the above named research study has been granted from the date of approval (see end of this notice) to the date it is submitted for assessment/examination.

2. APPROVED, BUT MINOR AMENDMENTS ARE REQUIRED BEFORE THE RESEARCH COMMENCES (see Minor Amendments box below): In this circumstance, re-submission of an ethics application is not required but the student must confirm with their supervisor that all minor amendments have been made before the research commences. Students are to do this by filling in the confirmation box below when all amendments have been attended to and emailing a copy of this decision notice to her/his supervisor for their records. The supervisor will then forward the student's confirmation to the School for its records.

3. NOT APPROVED, MAJOR AMENDMENTS AND RE-SUBMISSION REQUIRED (see Major Amendments box below): In this circumstance, a 
revised ethics application must be submitted and approved before any research takes place. The revised application will be reviewed by the same reviewer. If in doubt, students should ask their supervisor for support in revising their ethics application.

\section{DECISION ON THE ABOVE-NAMED PROPOSED RESEARCH STUDY}

(Please indicate the decision according to one of the 3 options above)

\section{APPROVED, BUT MINOR AMENDMENTS ARE REQUIRED BEFORE THE RESEARCH COM MENCES}

M inor amendments required (for reviewer):

Before starting the study the applicant should obtain the ethical clearance from other ethics committees as reported in section 4.1 of the application.

M ajor amendments required(for reviewer): 
Confirmation of making the above minor ame ndments (for students):

I have noted and made all the required minor amendments, as stated above, before starting my research and collecting data.

Student's name (Typed name to act as signature):

Student number:

Date:

(Please submit a copy of this decision letter to your supervisor with this box completed, if minor amendments to your ethics application are required)

\section{ASSESSMENT OF RISK TO RESEACHER (for reviewer)}

If the proposed research could expose the researcher to any of kind of emotional, physical or health and safety hazard? Please rate the degree of risk:

$\mathrm{HIGH}$

MEDIUM 
Reviewer comments in relation to researcher risk (if any):

Reviewer(Typed name to act as signature): $\quad$ Matteo Martini

Date: $25 / 11 / 2015$

This reviewer has assessed the ethics application for the named research study on behalf of the School of Psychology Research Ethics Committee

\section{PLEASE NOTE:}

*For the researcher and participants involved in the above named study to be covered by UEL's insurance and indemnity policy, prior ethics approval from the School of Psychology (acting on behalf of the UEL Research Ethics Committee), and confirmation from students where minor amendments were required, must be obtained before any research takes place.

*For the researcher and participants involved in the above named study to be covered by UEL's insurance and indemnity policy, travel approval from UEL (not the School of Psychology) must be gained if a researcher intends to travel overseas to collect data, even if this involves the researcher travelling to his/her home country to conduct the research. Application details can be found here:

http://www.uel.ac.uk/gradschool/ethics/fieldwork/ 


$$
\text { APPENDIX F - Change of Title } 1
$$

\section{Change proiect title - Miss Natalie Bailey}

The Psychology Research Degrees Sub-Committee on behalf of the University Quality and Standards Committee has considered your request. The decision is:

\section{Approved}

Your new thesis title is confirmed as follows:

Old thesis title: How do older adults in the UK's Black Caribbean Community make sense of copying with depression in later life? A qualitative study on help-seeking attitudes.

New thesis title: Coping with depression in later life: A qualitative study of help-seeking attitudes among Older Black Caribbean adults in the UK.

Your registration period remains unchanged.

Thesis title updated again as requested by viva examiners to

A qualitative study to explore the help seeking views relating to depression among older Black Caribbean adults living in the UK 
APPENDIX G - Example of Emerging Themes Process

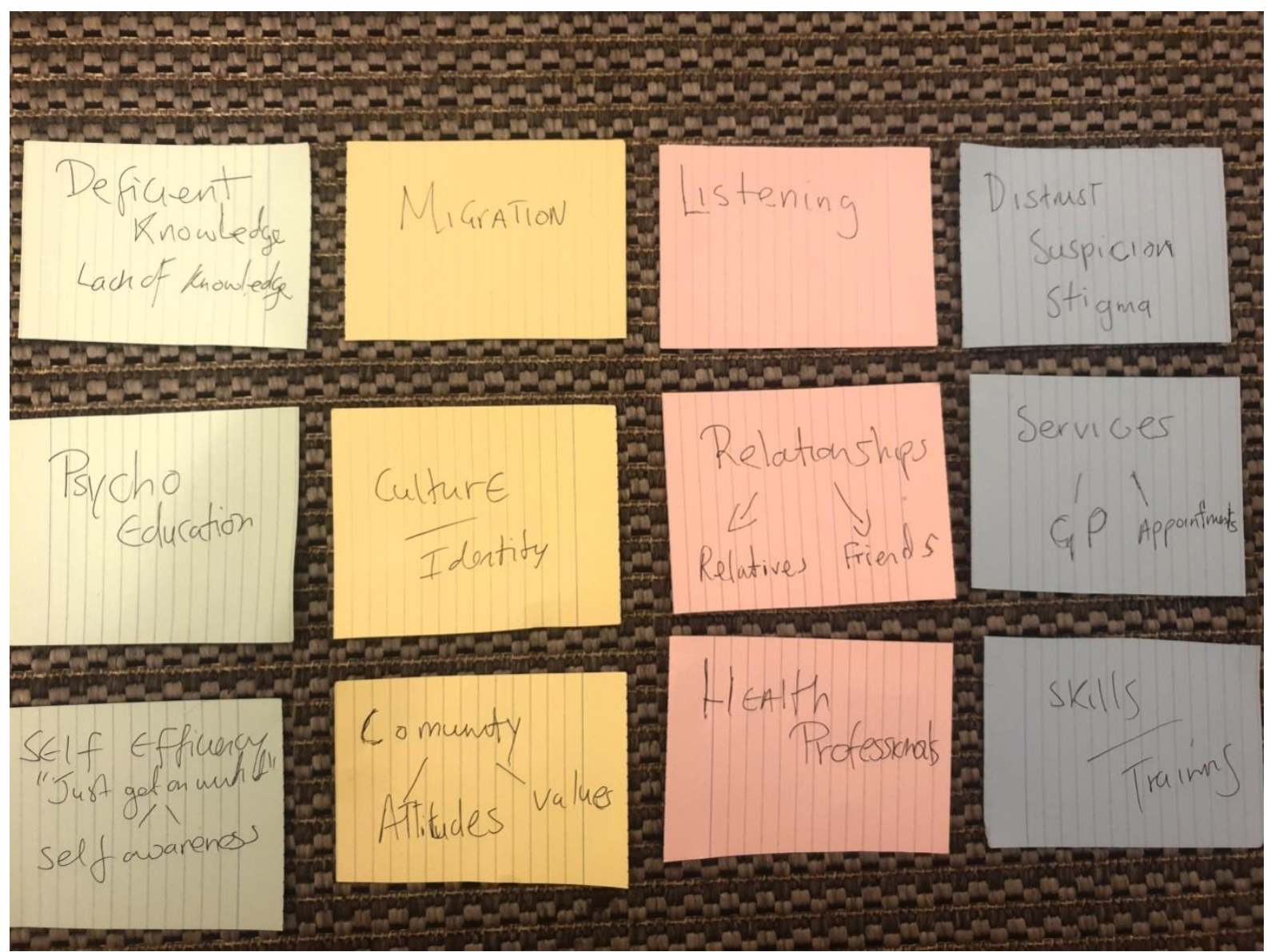


APPENDIX H - Examination of Data Process Example 1

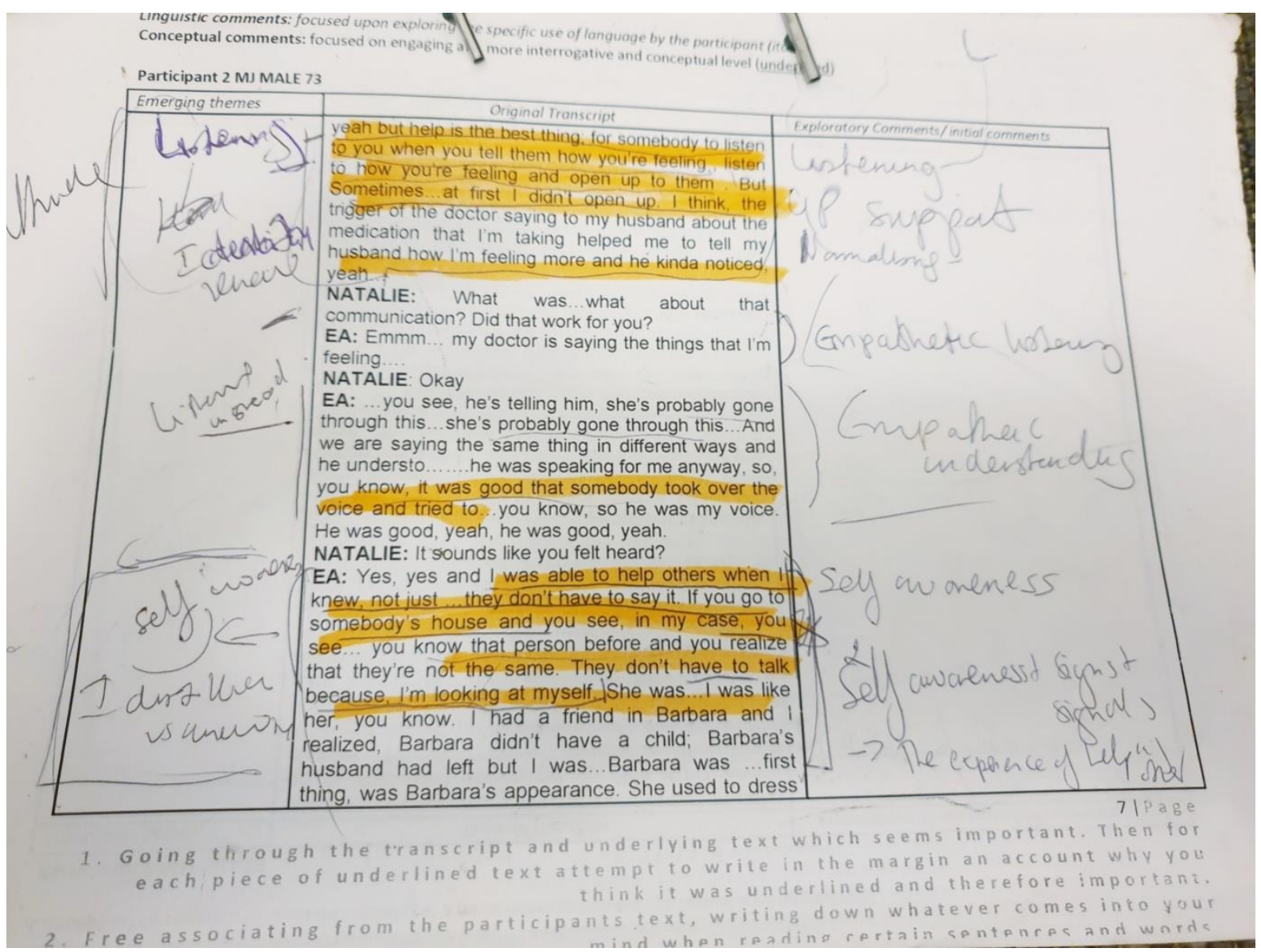


APPENDIX I - Examination of Data Process Example 2

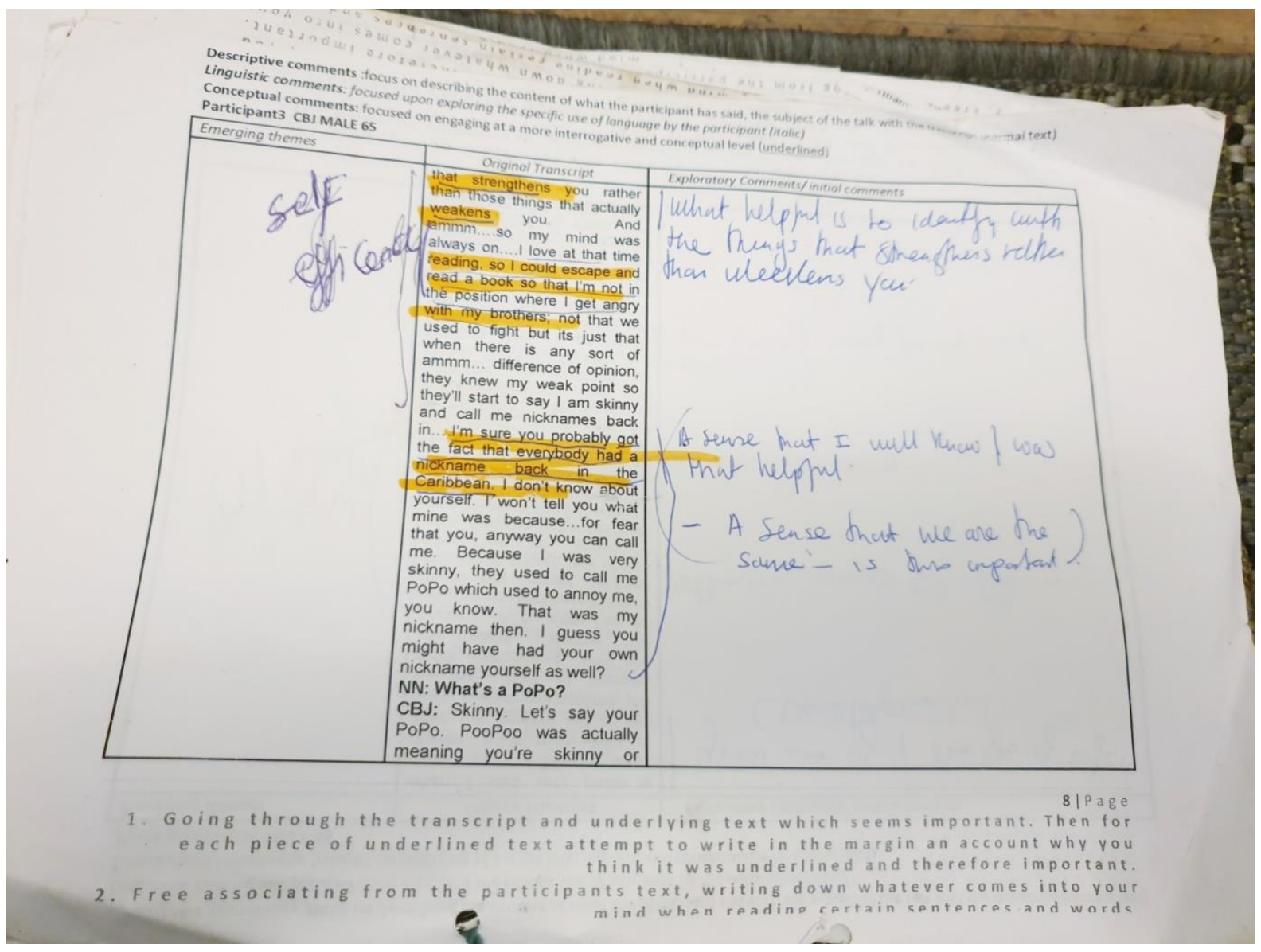


APPENDIX J - Examination of Data Process Example 3

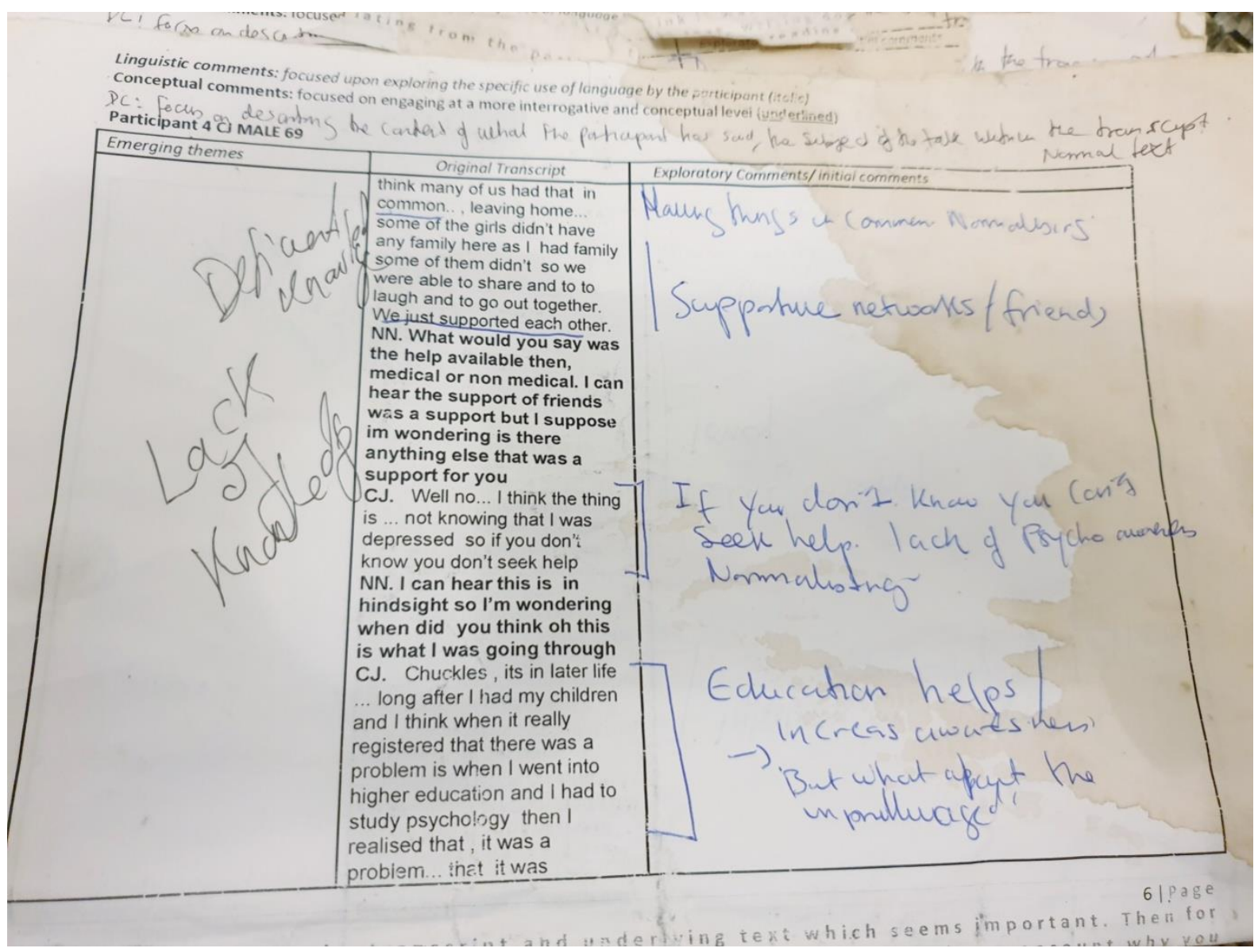




\section{APPENDIX K - Examination of Data Process Example 4}

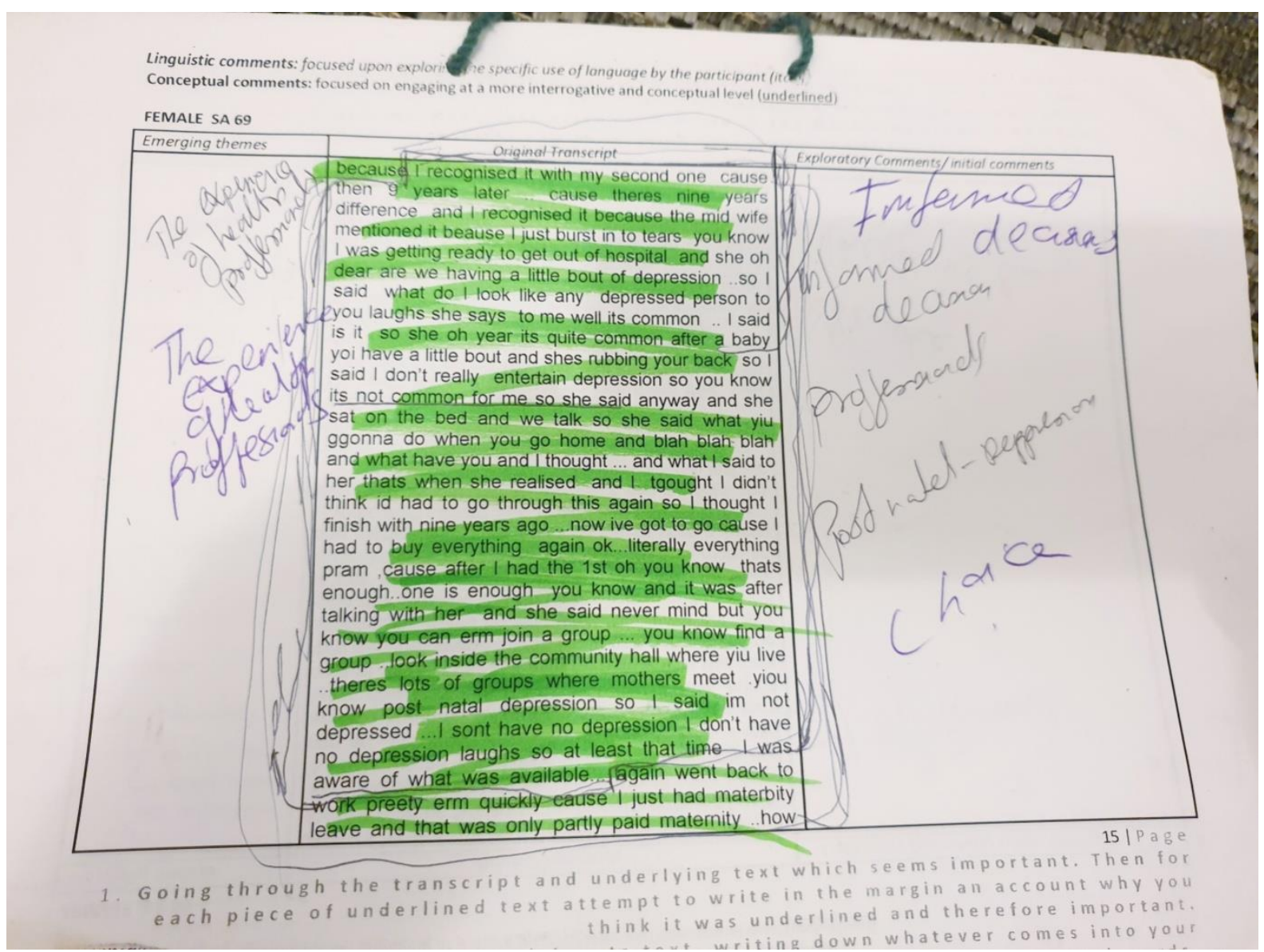

